\title{
RELATIVE DING STABILITY AND AN OBSTRUCTION TO THE EXISTENCE OF MABUCHI SOLITONS
}

YI YAO

\begin{abstract}
Авstract. Mabuchi solitons generalize Kähler-Einstein metrics on Fano manifolds, which constitute a Yau-Tian-Donaldson type correspondence with relative Ding stability. Comparing with Kähler-Ricci solitons, there is a distinct necessary condition for the existence. We show this condition can be implied by the uniformly relative Ding stability. For this we study the inner product of $\mathbb{C}^{*}$-actions on equivariant test-configurations and obtain an integration formula over the total space. To analyze the uniform stability, by adapting Okounkov body construction to the setting of torus action, we give a convex-geometry description for the reduced non-Archimedean J-functionals.
\end{abstract}

\section{INTRODUCTION}

Since the Yau-Tian-Donaldson correspondence for Fano manifolds is established, namely the equivalence between the existence of Kähler-Einstein (KE) metrics and the K-polystability of Fano manifolds, it is desired to extend this correspondence to other kinds of canonical metrics. It is well-known that a Fano manifold $M$ with nonvanishing Futaki invariant does not admit KE metrics, we can seek other canonical metrics such as Kähler-Ricci(KR) solitons and extremal metrics. In [30], Mabuchi introduced a new kind of generalized KE metrics now we call Mabuchi solitons, since they give self-similar solutions of the gradient flow of Ding functional, see [11]. Let

$$
\omega=\sqrt{-1} g_{i \bar{j}} d z^{i} \wedge d \bar{z}^{j} \in 2 \pi c_{1}(M)
$$

be a Kähler metric with Ricci potential $h_{\omega}$ which satisfies $\operatorname{Ric}(\omega)-\omega=i \partial \bar{\partial} h_{\omega}$ and $\int e^{h_{\omega}} \omega^{n}=V:=\int \omega^{n}$. By the definition in [30], $\omega$ is called a Mabuchi soliton if the $(1,0)$-gradient vector field

$$
\operatorname{grad}_{\omega} e^{h_{\omega}}:=\sum_{i, j}\left(g^{i \bar{j}} \frac{\partial e^{h_{\omega}}}{\partial \bar{z}^{j}}\right) \frac{\partial}{\partial z^{i}}
$$

is holomorphic. It turns out this vector field must coincide with the extremal vector field $Z$ defined in [15], which can be determined in advance by Futaki invariant and a chosen maximal compact subgroup $K$ of $\operatorname{Aut}^{0}(M)$. Mabuchi solitons can be regarded as an algebraic analogue of KR-solitons which require $\operatorname{grad}_{\omega} h_{\omega}$ is holomorphic. From the view of PDE, fixing a background metric $\omega_{0}$, let $\theta_{0}$ be the Hamiltonian function of $Z$ w.r.t. $\omega_{0}$ which satisfies $\iota_{Z} \omega_{0}=i \bar{\partial} \theta_{0}$ and $\int \theta_{0} \omega_{0}^{n}=0$. Then $\omega_{u}=\omega_{0}+i \partial \bar{\partial} u$ is a Mabuchi soliton if and only if $u$ satisfies a Monge-Ampère type equation

$$
\left(1-\theta_{Z}(u)\right) \omega_{u}^{n}=e^{h_{\omega_{0}}-u} \omega_{0}^{n},
$$

2020 Mathematics Subject Classification. 32Q15, 32Q20, 14D06.

Key words and phrases. Generalized Kähler-Einstein metrics, Mabuchi solitons, Equivariant test configurations, Ding stability, Okounkov body. 
where $\theta_{Z}(u)=\theta_{0}+Z(u)$ is the Hamiltonian function of $Z$ w.r.t. $\omega_{u}$, satisfying $\iota_{Z} \omega_{u}=$ $i \bar{\partial} \theta_{Z}(u)$ and $\int \theta_{Z}(u) \omega_{u}^{n}=0$. As a comparison, a KR-soliton $\omega_{u}$ should satisfy

$$
\omega_{u}^{n}=e^{h_{\omega_{0}}-\theta_{X}(u)-u} \omega_{0}^{n},
$$

where $X$ is a holomorphic vector field. A significant difference is that (1.1) admits a smooth solution only if

$$
\vartheta(M):=\max _{M} \theta_{Z}(u)<1 .
$$

Actually, this maximum is an invariant of Fano manifold $M$ (see Def. 5.4 for details, we alter the notation $\alpha_{M}$ used in [30] to avoid confusion with Tian's $\alpha$-invariants). It concerns with the Duistermaat-Heckman measure of the extremal action, i.e. the $\mathbb{C}^{*}$-action generated by $Z$.

Mabuchi solitons also can be regarded as an analogue of extremal metrics from the view of a new GIT (geometric invariant theory) model recently constructed by Donaldson [14], in which the moment map is Ricci potential $1-e^{h_{\omega}}$, contrasting to scalar curvature in the old model. The stability notion in the new model is Ding stability (abbr. as D-stability in below) which arised from the study of limit slopes of Ding functional by Berman [3]. As with extremal metrics are critical points of Calabi's energy, Mabuchi solitons are critical points of a similar functional

$$
\operatorname{Din}(\omega)=\frac{1}{V} \int\left(1-e^{h_{\omega}}\right)^{2} \omega^{n}, \omega \in 2 \pi c_{1}(M)
$$

called Ding energy, see [42]. Hence from the view of a general framework in [39], as with extremal metrics correspond to relative K-stability, Mabuchi solitons should correspond to the relative version of D-stability.

When $M$ is a toric manifold, in [42], relative D-stability is defined in terms of limit slopes of the modified Ding functional along toric geodesic rays. In this situation, (uniformly) relative D-stable is equivalent to $\vartheta(M) \leq 1(\vartheta(M)<1)$, and $M$ admits a Mabuchi soliton if and only if it is uniformly relative D-stable.

Relative D-stability is extended to general Fano manifolds in [11,23]. Let $T \subset \operatorname{Aut}^{0}(M)$ be a torus complexifying real torus $S$. Taking a maximal compact subgroup $K \subset \operatorname{Aut}^{0}(M)$ containing $S$, which determines an extremal vector field $Z$. Let

$$
\mathcal{H}_{\omega}^{Z}=\left\{u \in C^{\infty}(M) \mid \omega_{u}>0, \operatorname{Im} Z . u=0\right\}
$$

be the space of $\operatorname{Im} Z$-invariant potentials. The modified Ding functional $D_{Z}$ is defined on $\mathcal{H}_{\omega}^{Z}$ and takes Mabuchi solitons as the critical points,

$$
D_{Z}(u):=-E_{Z}(u)-\log \left(\frac{1}{V} \int_{M} e^{h_{\omega}-u} \omega^{n}\right),
$$

see Definition 5.7 for $E_{Z}$. Follow the work [3] of Berman, for a $T$-equivariant test-configurations $(\mathcal{X}, \mathcal{L})$ of $\left(M,-K_{M}\right)$, consider the limit slope of $D_{Z}$ along the associated Phong-Sturm's geodesic ray. The result is called the relative Berman-Ding invariant:

$$
D_{Z}^{N A}(\mathcal{X}, \mathcal{L})=D^{N A}(\mathcal{X}, \mathcal{L})+\left\langle\alpha, \beta_{Z}\right\rangle,
$$

where $D^{N A}$ is Berman-Ding invariant (2.5) and $\left\langle\alpha, \beta_{Z}\right\rangle$ is the inner product of two $\mathbb{C}^{*}$ actions on $(\mathcal{X}, \mathcal{L})$ which will be discussed in below.

Then $M$ is said to be $D$-semistable relative to $T$ if

$$
D_{Z}^{N A}(\mathcal{X}, \mathcal{L}) \geq 0
$$


for all $T$-equivariant test-configurations $(\mathcal{X}, \mathcal{L}) . M$ is said to be uniformly $\mathrm{D}$-stable relative to $T$ if there exists $\delta>0$ such that

$$
D_{Z}^{N A}(\mathcal{X}, \mathcal{L}) \geq \delta \cdot J_{T}^{N A}(\mathcal{X}, \mathcal{L})
$$

for all $T$-equivariant test-configurations, where $J_{T}^{N A}$ is the reduced non-Archimedean(NA) $J$-functional introduced by Hisamoto [22].

In [29], it shows that the existence of Mabuchi solitons is equivalent to the properness of $D_{Z}$. Then existence can imply the above uniform stability by taking the limit slopes. For the converse direction, Hisamoto [23] and Han-Li [16] shows that uniform stability and $\vartheta(M)<1$ can imply the existence, based on the variational approach of [4]. Han-Li's work deals with more general $g$-solitons, including Mabuchi solitons and KR-solitons.

With the expectation that all obstructions to the existence of canonical metrics could be explained as some stability conditions, our main result is that the additional assumption $\vartheta(M)<1$ can implied by the uniform stability condition. This is easily obtained in the toric case [42]. More specifically, we have

Theorem 1.1 (Main Theorem, see Theorem 6.1, 8.1). Let $M$ be a Fano manifold, $T \subset$ $A u t^{0}(M)$ be a torus. If $M$ is D-semistable relative to $T$ in the sense of $(1.2)$, then $\vartheta(M) \leq 1$. Furthermore, if $M$ is uniformly $D$-stable relative to $T$ in the sense of (1.3), then

$$
\vartheta(M) \leq 1-\delta<1
$$

Comparing with K-stability, a new feature of relative D-stability is that uniformly stability may be not equivalent to stability. At least there is an orbifold toric surfaces (see Example 5.14 [42]) which is relative D-stable but not uniformly, but the existence of smooth such examples is not known yet.

In the following, we outline the proof of Theorem 1.1 and introduce its byproducts which may benefit the study of relative K-stability.

We construct a specific family of $T$-equivariant test-configurations $\left(\mathcal{X}, \mathcal{L}_{c}\right)$ with a parameter $c$, that is deformation to the normal cone of a $T$-fixed point. Then we need to analyze the relative Berman-Ding invariant,

$$
D_{Z}^{N A}\left(\mathcal{X}, \mathcal{L}_{c}\right)=D^{N A}\left(\mathcal{X}, \mathcal{L}_{c}\right)+\left\langle\alpha, \beta_{Z}\right\rangle_{0} .
$$

The first term (Berman-Ding invariant) is easy to compute, so we focus on $\left\langle\alpha, \beta_{Z}\right\rangle_{0}$, which is the inner product of the structure $\mathbb{C}^{*}$-action $\alpha$ on $\left(\mathcal{X}, \mathcal{L}_{c}\right)$ and the fiberwise extremal action $\beta_{Z}$. We introduce some new methods to deal with it.

Inner products of $\mathbb{C}^{*}$-actions. Let $(M, L)$ be a polarized manifold with a lifted action $\beta: \mathbb{C}^{*} \rightarrow \operatorname{Aut}(M, L)$ generated by vector field $X$. Let $(\mathcal{X}, \mathcal{L})$ be a $\mathbb{C}^{*}$-equivariant testconfiguration for $(M, L)$ with structure action $\alpha$ and fiberwise action $\beta$. In the following, $\mathcal{X}$ always means the canonically compactified family over $\mathbb{P}^{1}$. The inner product $\langle\alpha, \beta\rangle_{0}$ is defined by Székelyhidi [39] via the induced $\mathbb{C}^{*}$-actions on $\mathrm{H}^{0}\left(\mathcal{X}_{0}, k \mathcal{L}_{0}\right)$, it requires $\mathcal{L}$ is relatively ample, see (3.1). In Section 3.2, we give a new definition, denoted by $\langle\alpha, \beta\rangle$ see (3.6), in terms of intersection numbers over the associated bundles for action $\beta$. Naturally, $\langle\alpha, \beta\rangle$ is invariant under pulling-back of test-configurations and valid for general $\mathcal{L}$. It coincides with $\langle\alpha, \beta\rangle_{0}$ when $\mathcal{L}$ is relatively ample, see Theorem 3.9.

This new definition gives rise to a new proof for the limit slope formula for the modified terms of energy functionals, e.g. modified K-energy (5.3) for extremal metrics and modified Ding functional (5.2) for Mabuchi solitons.

Theorem 1.2 (Limit slopes of the modified terms, see Theorem 3.7). Let $(\mathcal{X}, \mathcal{L})$ be a $\mathbb{C}^{*}$-equivariant test-configuration for $(M, L)$, where $\mathcal{L}$ is not necessarily ample. Let $\Phi$ be 
a $\alpha\left(\mathbb{S}^{1}\right) \times \beta\left(\mathbb{S}^{1}\right)$-invariant metric on $\mathcal{L}$ satisfying condition $\boldsymbol{A}$ or $\boldsymbol{B}$ in Definition 3.3. It induces a ray of metrics $\left\{u_{t}\right\}_{t \geq 0}$ on $L$ with curvature form $\omega_{u_{t}}$. Suppose the fiberwise action $\beta$ is generated by vector field $X$ on $M$, which has normalized Hamiltonian function $\theta_{X}\left(u_{t}\right)$ with respect to $\omega_{u_{t}}$. Then we have

$$
\lim _{t \rightarrow+\infty} \frac{1}{V} \int_{M} \dot{u}_{t} \theta_{X}\left(u_{t}\right) \omega_{u_{t}}^{n}=\langle\alpha, \beta\rangle .
$$

Case $\mathbf{A}$ includes any smooth metric $\Phi$, not necessarily having positive curvature (even along fibers). When $\mathcal{L}$ is ample and $\left\{u_{t}\right\}$ is Phong-Sturm's geodesic ray (contained in case B), this have been obtained by Hisamoto [21] through approximation by Bergman geodesics.

Our method uses equivariant Hirzebruch-Riemann-Roch (HRR) formula, so it is valid for general rays of metrics. It is similar to the method used by Donaldson [13] (Proposition 3 ) when dealing with the norm $\|(\mathcal{X}, \mathcal{L})\|_{2}$. Firstly, by Stokes formula, we convert the limit in (1.4) to an integral of Hamiltonian function of $X$ over the total space $\mathcal{X}$. Then we apply equivariant HRR formula on $\mathcal{X}$, it relates the integral to the leading coefficient of equivariant Euler characteristic $\chi_{1}^{\beta}(\mathcal{X}, k \mathcal{L})$ (Definition 3.6). Finally, via Leray's spectral sequences, $\chi_{1}^{\beta}$ can be further related to $\langle\alpha, \beta\rangle$. There is a little trouble is that we need smooth total space $\mathcal{X}$ to apply equivariant HRR, but thanks to the pulling-back invariance of $\langle\alpha, \beta\rangle$, we can apply HRR on a resolution of $\mathcal{X}$ and then come back.

As a byproduct, we obtain an integral formula for inner products.

Theorem 1.3 (Integral formula for inner products, see Theorem 3.7 (2)). Assume the total space $\mathcal{X}$ is smooth. We also denote by $X$ the generating vector field on $\mathcal{X}$ for the fiberwise action $\beta$. Given a smooth 2 -form $\Omega \in 2 \pi c_{1}(\mathcal{L})$ and function $\Theta$ on $\mathcal{X}$ satisfying $\iota_{X} \Omega=i \bar{\partial} \Theta$ and $\int_{\mathcal{X}_{1}} \Theta \Omega^{n}=0$ (equivalent to integrate along any smooth fiber), then we have

$$
\langle\alpha, \beta\rangle=\frac{1}{(n+1) L^{n}} \int_{\mathcal{X}} \Theta\left(\frac{\Omega}{2 \pi}\right)^{n+1}=\text { const } \cdot \int_{\mathcal{X}}(\Theta+\Omega)^{n+2} .
$$

Note the middle integral only involves the Hamiltonian function for action $\beta$. The rightmost formula hints us to compute $\langle\alpha, \beta\rangle$ by localization method on $\mathcal{X}$.

Go back to the proof of Theorem 1.1. Firstly, by (1.5) we express $\left\langle\alpha, \beta_{Z}\right\rangle$ as an integral over $\mathcal{X}$, then expand it in terms of $c$. After evaluating the integrals in the coefficients (by a localization argument), we found

$$
D_{Z}^{N A}\left(\mathcal{X}, \mathcal{L}_{c}\right)=\frac{1-\vartheta(M)}{(n+1) c_{1}(M)^{n}} c^{n+1}+A c^{n+2}, 0<c \ll 1,
$$

where $A$ is a constant independent of $c$. By this expansion, relative D-semistability immediately implies $\vartheta(M) \leq 1$. But for the case of uniform stability, we need to expand $J_{T}^{N A}\left(\mathcal{X}, \mathcal{L}_{c}\right)$ in terms of $c$. This leads us to study the reduced non-Archimedean J-functionals.

A convex-geometry description for the reduced non-Archimedean J-functionals. Let $(\mathcal{X}, \mathcal{L})$ be a $T$-equivariant test-configuration, the reduced non-Archimedean(NA) J-functional $J_{T}^{N A}(\mathcal{X}, \mathcal{L})$ was introduced by Hisamoto [22]. It is defined as the infimum of values of NA J-functional $J^{N A}$ on the all twistings of $(\mathcal{X}, \mathcal{L})$ by 1-parameter subgroups $\rho: \mathbb{C}^{*} \rightarrow T$, see Definition 7.1 for the twisting operation.

By [41], we know $(\mathcal{X}, \mathcal{L})$ induces a filtration $\mathcal{F}(\mathcal{X}, \mathcal{L})$ on the section ring $R(M, L)$, see Section 4.3. It is more natural to twist $\mathcal{F}(\mathcal{X}, \mathcal{L})$, since this also makes sense for irrational $\rho$. We denotes by $\mathcal{F}(\mathcal{X}, \mathcal{L})^{\rho}(7.2)$ the twisted filtration, then $J_{T}^{N A}$ is defined by

$$
J_{T}^{N A}(\mathcal{X}, \mathcal{L}):=\inf _{\rho \in \mathbb{R}^{m}} J^{N A}\left(\mathcal{F}(\mathcal{X}, \mathcal{L})^{\rho}\right)
$$




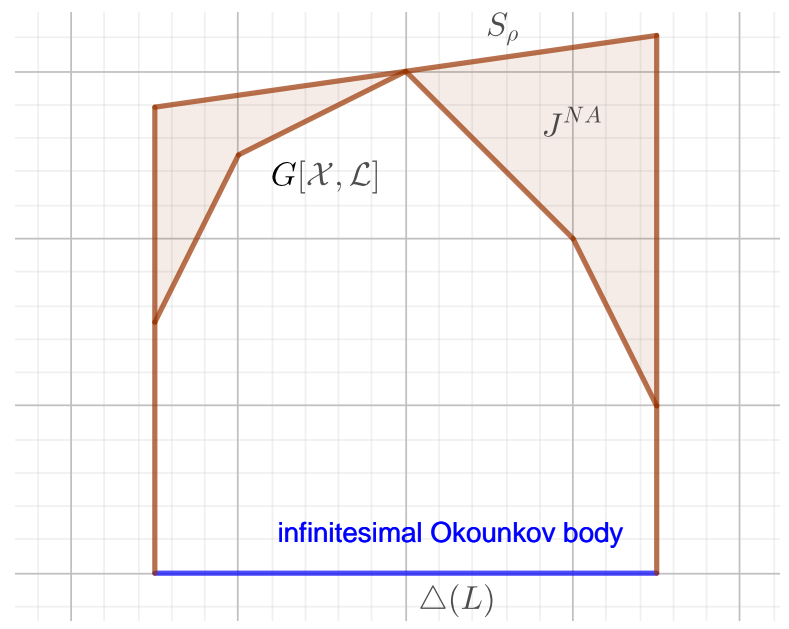

FIGURE 1.1. The shaded area is $J^{N A}$

see (7.3) for evaluating $J^{N A}$ on filtrations. When $(\mathcal{X}, \mathcal{L})$ dominates the trivial product, $J^{N A}$ can be expressed as intersection numbers over $\mathcal{X}$, see (2.4). But when we twist $(\mathcal{X}, \mathcal{L})$, it no longer dominates the product. Thus we need another method to analyze $J_{T}^{N A}$.

When $(M, L)$ is the toric manifold associated to Delzant polytope $P$, and $(\mathcal{X}, \mathcal{L})$ is a toric test-configuration associated to a piecewise linear concave function $g$, Hisamoto [22] gives an explicit formula

$$
J_{T}^{N A}(\mathcal{X}, \mathcal{L})=\inf _{\ell} \frac{1}{|P|} \int_{P}\left(\sup _{P}(g+\ell)-(g+\ell)\right) d y
$$

where $\ell$ runs over all affine functions. It is same to the minimum volume of the region bounded by the graph of $g$ and its support function, see the shadow area in Fig. 1.1.

We extend this picture to general setting by replacing $P$ with the Okounkov body $\triangle(L)$ [35]. By [6], filtration $\mathcal{F}(\mathcal{X}, \mathcal{L})$ induces a concave function $G[\mathcal{X}, \mathcal{L}]$ on $\triangle(L)$ called the concave transform of $\mathcal{F}(\mathcal{X}, \mathcal{L})$, which takes over the role of $g$ in the toric setting. But for adapting with the $T$-action, we employ the infinitesimal Okounkov body introduced by Lazarsfeld-Mustaţă [26], which is associated to a flag of subspaces of the tangent space at a $T$-fixed point. This construction gives rise to a $\mathbb{R}^{m}$-valued affine function $H_{\beta}$ on $\triangle(L)$, decided by $T$-action around the fixed point, see (7.11).

Theorem 1.4 (A convex-geometry description for $J_{T}^{N A}$ ). Let $T \subset$ Aut $(M, L)$ be a torus, and $(\mathcal{X}, \mathcal{L})$ a T-equivariant ample test-configuration for $(M, L)$. Let $\triangle(L)$ be the infinitesimal Okounkov body (Definition 7.8) associated to a $T$-fixed point and an infinitesimal flag. Let $G=G[\mathcal{X}, \mathcal{L}]$ be the concave transform (see Theorem 7.11) of the associated filtration $\mathcal{F}(\mathcal{X}, \mathcal{L})$ on the section ring.

(1) For any $\rho \in \mathbb{R}^{m}$, the concave transform of the twisted filtration $\mathcal{F}(\mathcal{X}, \mathcal{L})^{\rho}(7.2)$ is $G+\left\langle\rho, H_{\beta}\right\rangle$. We have

$$
J^{N A}\left(\mathcal{F}(\mathcal{X}, \mathcal{L})^{\rho}\right)=\sup _{\triangle(L)}\left(G+\left\langle\rho, H_{\beta}\right\rangle\right)-\int_{\triangle(L)}\left(G+\left\langle\rho, H_{\beta}\right\rangle\right) \frac{d x}{|\triangle(L)|},
$$

and $J_{T}^{N A}(\mathcal{X}, \mathcal{L})$ is the infimum of above quantities over $\rho \in \mathbb{R}^{m}$. 
(2) Let $c$ be the constant making the affine function $S_{\rho}:=c-\left\langle\rho, H_{\beta}\right\rangle$ to be a support function of $G$, i.e. $\inf _{\Delta}\left(S_{\rho}-G\right)=0$, then

$$
J^{N A}\left(\mathcal{F}(\mathcal{X}, \mathcal{L})^{\rho}\right)=\int_{\triangle(L)}\left(S_{\rho}-G\right) \frac{d x}{|\triangle(L)|},
$$

which is the shadow area in Fig. 1.1. In particular, $J_{T}^{N A}(\mathcal{X}, \mathcal{L})$ is bounded below by the infimum of above quantities when $S_{\rho}$ running over all support functions of $G$.

Go back to the proof of Theorem 1.1, with above convex-geometry description of $J_{T}^{N A}$, we can show

$$
J_{T}^{N A}\left(\mathcal{X}, \mathcal{L}_{c}\right)=\frac{c^{n+1}}{(n+1) L^{n}}, \text { when } c \ll 1 .
$$

Combing with (1.6), we see uniformly relative D-stability implies $\vartheta(M)<1$. The proof of main theorem is completed.

From Monge-Ampère measures to Duistermaat-Heckman measures. Let $\left\{u_{t}\right\}$ be the PhongSturm's geodesic ray associated to a test-configuration $(\mathcal{X}, \mathcal{L})$, Hisamoto [20] showed the pushforward of Monge-Ampère measure $V^{-1} \omega_{u_{t}}^{n}$ by $\dot{u}_{t}$ is equal to the Duistermaat-Heckman measure $\operatorname{DH}(\mathcal{X}, \mathcal{L})$, see Section 2.2 for definition. In particular, these pushforward measures are independent of $t$, but this no longer holds for general rays. By similar techniques with the proof of (1.4), we extend Hisamoto's result to a more wider class of rays.

Theorem 1.5. Let $(\mathcal{X}, \mathcal{L})$ be an ample test-configuration for $(M, L)$. Let $\Phi$ be a $\alpha\left(\mathbb{S}^{1}\right)$ invariant metric on $\mathcal{L}$ satisfying condition $\boldsymbol{A}$ or $\boldsymbol{B}$ in Definition 3.3. It induces a ray of metrics $\left\{\phi_{t}=\psi_{0}+u_{t}\right\}_{t \geq 0}$ on L. We assume $\omega_{u_{t}} \geq 0$ in case $\boldsymbol{A}$. Then the pushforward measure

weakly when $t \rightarrow+\infty$.

$$
\left(\dot{u}_{t}\right)_{\#}\left(\frac{1}{V} \omega_{u_{t}}^{n}\right) \rightarrow \operatorname{DH}(\mathcal{X}, \mathcal{L})
$$

Kähler-Ricci solitons, Mabuchi solitons and extremal metrics. For Fano manifolds, there are three kinds of canonical metrics in $c_{1}(M)$ listed in the title (abbr. KRS, MS and extK). KRS and MS are studied as instances of more general $g$-solitons in [16], where contains a complete discussion of existence, stability and properness of energy functionals. For extK (in general class), the equivalence of existence with properness of modified K-energy has been established in [9], also see [19]. From properness to uniform stability can be obtained by the limit slope formulas in $[28,16]$. The reverse direction has not been fully achieved, see [28] for recent progress via BBJ's [4] approach.

It seems that KRS has no direct connection to MS except they satisfy similar equations. Toric manifolds always admit KRS [40] but not for MS [42]. Although MS and extK satisfy very different equation (second v.s. fourth order), but the existence of MS can imply the existence of extK due to inequality: $M_{Z} \geq D_{Z}-C$, see Remark 5.8.

Some of related works. For a $\mathbb{C}^{*}$-equivariant test-configuration $(\mathcal{X}, \mathcal{L})$ with smooth $\mathcal{X}$, let $\Theta_{W}$ and $\tilde{\Theta}_{X}$ be the Hamiltonian function on $\mathcal{X}$ for action $\alpha$ and $\beta$ respectively. In [12], $\langle\alpha, \beta\rangle_{0}$ is defined to be $\int_{\mathcal{X}_{0}} \Theta_{W} \tilde{\Theta}_{X} \Omega^{n}$, then (1.4) is obtained easily. Our method avoids to integrate along $\mathcal{X}_{0}$, since it may be singular and nonreduced. Instead, formula (1.5) integrating over $\mathcal{X}$ which can be smooth after taking a resolution. We also can use (1.5) to define inner products of actions on test-configurations for general Kähler manifolds in the sense of [12]. This method that converting limit slopes to an integral over $\mathcal{X}$ had been employed by Sjöström Dyrefelt [38] to extend K-stability to transcendental Kähler classes. Finally, the twisting of filtrations is also introduced in the work of $\mathrm{Li} \mathrm{[27].}$ 
Organization. In Section 2, we review main notions and tools, including test-configurations, Hamiltonian functions and equivariant HRR formula. In Section 3, we study inner products of $\mathbb{C}^{*}$-actions. In Section 4, we prove Theorem 1.5 (this section can be skipped if the reader only cares the main theorem). In Section 5, we review Mabuchi solitons, energy functionals and relative D-semistability. In Section 6, we show semistability implies $\vartheta(M) \leq 1$. In Section 7, we review the twisting of test-configurations (filtrations), $J_{T}^{N A}$ and uniformly relative D-stability, and give a convex-geometry description for $J_{T}^{N A}$ via infinitesimal Okounkov body. Finally, in Section 8, we show uniform stability implies $\vartheta(M)<1$.

Conventions and notations. Let $V$ be a vector space with a $\mathbb{C}^{*}$-action. For $\tau \in \mathbb{C}^{*}$ and $x \in V, \tau . x$ denotes the action. We denote by $V_{\mu}:=\left\{x \in V \mid \tau . x=\tau^{\mu} x\right\}$ the weight subspace for each weight $\mu \in \mathbb{Z}$. We use $\mu$ to denote the weights of action $\alpha$ and $\nu$ for action $\beta$. If $V$ carries two commuting $\mathbb{C}^{*}$-action $\alpha$ and $\beta$, then it carries a $\mathbb{C}^{*} \times \mathbb{C}^{*}$-action denoted by $\alpha \times \beta$. We denote by $V_{\mu, \nu}$ the weight subspace with weight $\mu$ and $\nu$ w.r.t. $\alpha$ and $\beta$ respectively.

Let $(M, L)$ be a polarized manifold, set $N_{k}=h^{0}(M, k L)=\frac{L^{n}}{n !} k^{n}+O\left(k^{n-1}\right)$. Suppose $h$ is a Hermitian metric on $L$ and $s$ is a local section of $L$, we usually use the local function $\phi=-\log |s|_{h}^{2}$ to denote $h$. Its curvature form is $\operatorname{Ric}(h)=i \partial \bar{\partial} \phi \in 2 \pi c_{1}(L)$. We set $V=\int(i \partial \bar{\partial} \phi)^{n}=(2 \pi)^{n} L^{n}$.

In this paper, a $G$-equivariant test-configuration $(\mathcal{X}, \mathcal{L})$ always means the canonically compactified family over $\mathbb{P}^{1}$. We use $\left.(\mathcal{X}, \mathcal{L})\right|_{\mathbb{C}}$ to denote the restricted family over $\mathbb{C}$ which is the original definition. The structure action is denoted by $\alpha: \mathbb{C}^{*} \rightarrow \operatorname{Aut}(\mathcal{X}, \mathcal{L})$, and the fiberwise action is denoted by $\beta: G \rightarrow \operatorname{Aut}(\mathcal{X}, \mathcal{L})$. The fibers are denoted by $\mathcal{X}_{\tau}:=$ $\pi^{-1}(\tau)$ and $\mathcal{L}_{\tau}:=\left.\mathcal{L}\right|_{\mathcal{X}_{\tau}}$ for $\tau=e^{-\frac{1}{2}(t+i s)} \in \mathbb{P}^{1}$.

\section{Preliminaries}

2.1. Test-configurations. Test-configurations abstracts the one-parameter subgroups in Hilbert-Mumford's criterion in geometric invariant theory.

Definition 2.1. Let $(M, L)$ be a polarized manifold. A test-configuration $(\mathcal{X}, \mathcal{L})$ for $(M, L)$ is constituted of a normal variety $\mathcal{X}$ with a $\mathbb{Q}$-line bundle $\mathcal{L}$ and

(1) a flat morphism $\pi: \mathcal{X} \rightarrow \mathbb{C}$;

(2) $a \mathbb{C}^{*}$-action $\alpha$ (called the structure action) on $\mathcal{X}$ such that $\pi$ is equivariant w.r.t. the multiplication action on $\mathbb{C}$;

(3) a lifting $\alpha: \mathbb{C}^{*} \rightarrow \operatorname{Aut}(\mathcal{X}, \mathcal{L})$, also called a linearization of $\alpha$;

(4) an isomorphism $\left(\mathcal{X}_{1}, \mathcal{L}_{1}\right) \cong(M, L)$.

We say $(\mathcal{X}, \mathcal{L})$ is (semi-)ample if $\mathcal{L}$ is relatively (semi-)ample w.r.t. $\pi$. Another testconfiguration $\left(\mathcal{X}^{\prime}, \mathcal{L}^{\prime}\right)$ is called a pullback of $(\mathcal{X}, \mathcal{L})$ if there is an equivariant birational map $f: \mathcal{X}^{\prime} \rightarrow \mathcal{X}$ which is isomorphism from $\mathcal{X}^{\prime} \backslash \mathcal{X}_{0}^{\prime}$ to $\mathcal{X} \backslash \mathcal{X}_{0}$ and $\mathcal{L}^{\prime}=f^{*} \mathcal{L}$. Following [7] Def. 6.1, if $\left(\mathcal{X}^{\prime}, \mathcal{L}^{\prime}\right)$ and $(\mathcal{X}, \mathcal{L})$ have a common pullback, we say they are equivalent with each other.

Let $G \subset \operatorname{Aut}(M, L)$ be a reductive subgroup, a G-equivariant test-configuration is a test-configuration $(\mathcal{X}, \mathcal{L})$ with a lifted $G$-action $\beta: G \rightarrow \operatorname{Aut}(\mathcal{X}, \mathcal{L})$ (called the fiberwise action) which preserves each fiber, commutes with $\alpha$ and coincides with $G \subset \operatorname{Aut}(M, L)$ on $\left(\mathcal{X}_{1}, \mathcal{L}_{1}\right) \cong(M, L)$.

Structure action $\alpha$ and isomorphism $\left(\mathcal{X}_{1}, \mathcal{L}_{1}\right) \simeq(M, L)$ induce an equivariant trivialization

$$
\left.(\mathcal{X}, \mathcal{L})\right|_{\mathbb{C}^{*}} \simeq\left(M \times \mathbb{C}^{*}, p_{1}^{*} L\right)
$$


where $\mathbb{C}^{*}$ trivially acts on the first factor of $M \times \mathbb{C}^{*}$. In particular, $\mathcal{X} \backslash \mathcal{X}_{0}$ is smooth. We can canonically compactify $(\mathcal{X}, \mathcal{L})$ by gluing it with the product $\left(M \times\left(\mathbb{P}^{1} \backslash\{0\}\right), p_{1}^{*} L\right)$ via the above trivialization. The result is a flat family over $\mathbb{P}^{1}$ with fibers are isomorphic to $(M, L)$ except over 0 . Note the action $\alpha$ on the fiber over $\infty$ is trivial. Since we almost always work on the compactified family, we use $(\mathcal{X}, \mathcal{L})$ to denote the compactified test-configurations, and use notation $\left.(\mathcal{X}, \mathcal{L})\right|_{\mathbb{C}}$ for the original family over $\mathbb{C}$.

Remark 2.2. If we change the lifted action $\alpha$ on $\left.\mathcal{L}\right|_{\mathbb{C}}$ by a character,

$$
\alpha(\tau) \rightsquigarrow \tau^{c} \cdot \alpha(\tau), \tau \in \mathbb{C}^{*}, c \in \mathbb{Z},
$$

then the compactified family $\mathcal{X}$ is unchanged, but $\mathcal{L}$ will change to $\mathcal{L}_{c}:=\mathcal{L} \otimes \pi^{*} \mathcal{O}_{\mathbb{P}^{1}}(c)$. The action $\alpha$ on $\mathcal{L}_{c}$ is the product of the original $\mathbb{C}^{*}$-action on $\mathcal{L}$ and the $\mathbb{C}^{*}$-action on $\mathcal{O}_{\mathbb{P}^{1}}(c)$. The action on $\mathcal{O}_{\mathbb{P}^{1}}(-1)$ is defined by

$$
\tau .\left(z_{0}, z_{1}\right)=\left(\tau^{-1} z_{0}, z_{1}\right), \text { for }\left.\left(z_{0}, z_{1}\right) \in \mathcal{O}_{\mathbb{P}^{1}}(-1)\right|_{\left[z_{0}, z_{1}\right]},
$$

note the action on fiber over $\infty=[0,1]$ is trivial.

2.2. Duistermaat-Heckman measures of test-configurations . Let $(\mathcal{X}, \mathcal{L})$ be an ample test-configuration for $(M, L)$, then the central fiber $\left(\mathcal{X}_{0}, \mathcal{L}_{0}\right)$ is a polarized scheme equipped with a $\mathbb{C}^{*}$-action. For sufficiently large and divisible $k$, consider the induced $\mathbb{C}^{*}$-action on $\mathrm{H}^{0}\left(\mathcal{X}_{0}, k \mathcal{L}_{0}\right)$, the weight distribution is defined by

$$
\mathfrak{m}_{k}:=\frac{1}{N_{k}} \sum_{\mu \in \mathbb{Z}} \operatorname{dim} \mathrm{H}^{0}\left(\mathcal{X}_{0}, k \mathcal{L}_{0}\right)_{\mu} \cdot \delta_{\mu / k} .
$$

By Corollary 3.4 in [7], $\left\{\mathfrak{m}_{k}\right\}$ have uniformly bounded support and converges weakly to a probability measure on $\mathbb{R}$, denoted by $\operatorname{DH}(\mathcal{X}, \mathcal{L})$, called the Duistermaat-Heckman $(D H)$ measure of $(\mathcal{X}, \mathcal{L})$.

In Section 4.3, $\mathrm{DH}(\mathcal{X}, \mathcal{L})$ is also equal to the limit measure of the associated filtration $\mathcal{F}(\mathcal{X}, \mathcal{L})$ of section ring. For a semiample test-configuration, its DH measure is defined to be that of its ample model, see Proposition 2.17 in [7] for details.

2.3. Non-Archimedean functionals. By the point of view of [7], a (equivalence class of) test-configuration $(\mathcal{X}, \mathcal{L})$ gives rise to a NA metric on the Berkovich analytification $\left(M^{a n}, L^{a n}\right)$. Many functionals in Kähler geometry have a NA counterpart which can be interpreted as the limit slopes along geodesic rays, see [8] for details.

(1) For any test-configuration $(\mathcal{X}, \mathcal{L})$ of $(M, L)$, the non-Archimedean Monge-Ampère energy is defined by

$$
E^{N A}(\mathcal{X}, \mathcal{L}):=\frac{\mathcal{L}^{n+1}}{(n+1) L^{n}},
$$

where $\mathcal{L}^{n+1}$ is the intersection number on $\mathcal{X}$. When $(\mathcal{X}, \mathcal{L})$ is semiample, Lemma 7.3 [7] says it equals to the barycenter of DH measure,

$$
E^{N A}(\mathcal{X}, \mathcal{L})=\int_{\mathbb{R}} \lambda d \operatorname{DH}(\mathcal{X}, \mathcal{L})
$$

(2) The non-Archimedean J-functional is defined by

$$
J^{N A}(\mathcal{X}, \mathcal{L}):=\sup \operatorname{suppDH}(\mathcal{X}, \mathcal{L})-\int_{\mathbb{R}} \lambda d \operatorname{DH}(\mathcal{X}, \mathcal{L}) .
$$


It can be expressed as intersection numbers. If $\mathcal{X}$ dominates the trivial product $M \times \mathbb{P}^{1}$, i.e. there exists $\Pi: \mathcal{X} \rightarrow M \times \mathbb{P}^{1}$ which is equivariant w.r.t. the trivial action on the target. Then we have

$$
J^{N A}(\mathcal{X}, \mathcal{L})=\left(L^{n}\right)^{-1} \mathcal{L} \cdot\left(\Pi^{*} p_{1}^{*} L\right)^{n}-E^{N A}(\mathcal{X}, \mathcal{L}) .
$$

(3) Suppose $M$ is a Fano manifold, $(\mathcal{X}, \mathcal{L})$ is an ample test-configuration for $\left(M,-K_{M}\right)$. There is a unique $\mathbb{Q}$-divisor $B$ supported on $\mathcal{X}_{0}$ such that

$$
\mathcal{L}+K_{\mathcal{X} / \mathbb{P}^{1}}=\mathcal{O}_{\mathcal{X}}(B)
$$

Let

$$
L^{N A}(\mathcal{X}, \mathcal{L}):=\operatorname{lct}\left(\mathcal{X},-B ; \mathcal{X}_{0}\right)-1
$$

where

$$
\operatorname{lct}\left(\mathcal{X},-B ; \mathcal{X}_{0}\right):=\sup \left\{t \in \mathbb{R} \mid \operatorname{pair}\left(\mathcal{X},-B+t \mathcal{X}_{0}\right) \text { is log canonical }\right\}
$$

is the log canonical threshold measured on $\mathcal{X}$. Then the Berman-Ding invariant (or nonArchimedean Ding functional) is defined as

$$
D^{N A}(\mathcal{X}, \mathcal{L}):=L^{N A}(\mathcal{X}, \mathcal{L})-E^{N A}(\mathcal{X}, \mathcal{L})
$$

By [3], it is the limit slope of classical Ding functional along the geodesic ray associated to $(\mathcal{X}, \mathcal{L})$.

2.4. Hamiltonian functions. Let $M$ be a Kähler manifold with a line bundle $L$ with Hermitian metric $h$. Its curvature form is $\omega=-i \partial \bar{\partial} \log h$. Let $X$ be a $(1,0)$ holomorphic vector field on $M$. A real-valued function $f$ is called a Hamiltonian function of $X$ w.r.t. $\omega$ if $\iota_{X} \omega=i \bar{\partial} f$. It implies $\mathcal{L}_{X} \omega=i \partial \bar{\partial} f$. Note that $f$ is unique up to a constant, and $\operatorname{Im} X$ preserves $\omega$. If further $\int f \omega^{n}=0$, we say $f$ is normalized and denote it by $\theta_{X}(\omega)$.

Suppose we have a lifted action $\gamma: \mathbb{C}^{*} \rightarrow \operatorname{Aut}(M, L)$ such that $\gamma\left(\mathbb{S}^{1}\right)$ preserves $h$. Let holomorphic vector field $X$ be the generator of $\gamma$ such that

$$
\gamma\left(e^{-\frac{1}{2}(t+i s)}\right)=\exp (t \cdot \operatorname{Re} X-s \cdot \operatorname{Im} X) .
$$

Note that it automatically implies $\exp (4 \pi \operatorname{Im} X)=i d_{M}$. We can obtain a Hamiltonian function of $X$ by taking derivative along the lifted action. Define

$$
f(x):=-\left.\frac{d}{d t}\left(\log \left|\gamma\left(e^{-\frac{t}{2}}\right) \cdot s\right|_{h}^{2}\right)\right|_{t=0}, x \in M, s \in L_{x} .
$$

It can be verified that $\iota_{X} \omega=i \bar{\partial} f$, and change the lifting $\gamma$ by a character will change $f$ by a constant. As usual, we use $\phi=-\log h$ to denote the Hermitian metric $h$, then the above formula can be shortly written as

$$
f:=\left.\frac{d}{d t}\left(\gamma\left(e^{-\frac{t}{2}}\right)^{*} \phi\right)\right|_{t=0} .
$$

We will call $f$ the Hamiltonian function induced by the lifted action $\gamma$, which is not necessarily normalized. 
2.5. Equivariant Hirzebruch-Riemann-Roch formula for line bundles. The equivariant HRR formula is our main tool to connect the integrals of Hamiltonian functions to the equivariant Euler characteristic. We only need the formula for equivariant line bundles, for its general versions, see [33] for the differential forms version and the appendix of [7] for the algebraic version.

Let $M$ be a $n$-dimensional complex manifold with a Hermitian line bundle $(L, h)$, where the curvature form $\omega=-i \partial \bar{\partial} \log h$ is not necessarily positive. There is a lifted action $\gamma: \mathbb{S}^{1} \rightarrow \operatorname{Aut}(M, L)$ preserving $h$, which is generated by holomorphic vector field $X$ such that $\gamma\left(e^{i t / 2}\right)=\exp (t \cdot \operatorname{Im} X)$. This lifting induces a Hamiltonian function $f$ for $X$ such that $\iota_{X} \omega=i \bar{\partial} f$.

Lifted action $\gamma$ induces $\mathbb{S}^{1}$-actions on cohomology groups $\mathrm{H}^{q}(M, k L)$. For $k \geq 1$, we define character function for $k L$ by

$$
\chi\left(k L, e^{i t}\right):=\sum_{q \geq 0}(-1)^{q} \operatorname{Tr}\left(e^{i t} \mid \mathrm{H}^{q}(k L)\right)=\sum_{q \geq 0}(-1)^{q} \sum_{\lambda \in \mathbb{Z}} e^{i \lambda t} \operatorname{dim} \mathrm{H}^{q}(k L)_{\lambda} .
$$

Then the equivariant HRR formula says

$$
\chi\left(k L, e^{i t}\right)=\int_{M} \operatorname{ch}\left(k L, h^{k}, t\right) \cdot \operatorname{td}(T M, g, t),
$$

where $g$ is any $\mathbb{S}^{1}$-invariant Hermitian metric on $T M$ (no relation with $\omega$ ), $\operatorname{ch}\left(k L, h^{k}, t\right):=$ $\exp k\left(\frac{\omega}{2 \pi}+i f t\right)$ is the equivariant Chern character form, and $\operatorname{td}(T M, g, t)$ is the equivariant Todd form whose zero degree part is 1 . Expanding both sides in terms of variable $t$, then comparing the coefficients of $t^{d}(d \geq 0)$, we obtain

$$
\sum_{q \geq 0}(-1)^{q} \sum_{\lambda \in \mathbb{Z}} \frac{\lambda^{d}}{d !} \operatorname{dim} \mathrm{H}^{q}(M, k L)_{\lambda}=\int_{M} \frac{f^{d}}{d !} \frac{(\omega / 2 \pi)^{n}}{n !} \cdot k^{n+d}+O\left(k^{n+d-1}\right) .
$$

What we really need in this paper is this expansion formula. The left hand side is called degree-d equivariant Euler characteristic, since when $d=0$ it is the classical Euler characteristic.

\section{INNER PRODUCT OF $\mathbb{C}^{*}$-ACTIONS ON A TEST-CONFIGURATION}

3.1. The original definition by Székelyhidi. We recall the inner product of $\mathbb{C}^{*}$-actions defined by Székelyhidi [39] in order to define relative K-stability. In this section, $(M, L)$ is a polarized manifold, and test-configurations are ample.

Let $(\mathcal{X}, \mathcal{L})$ be a $\mathbb{C}^{*}$-equivariant test-configuration for $(M, L)$ with structure action $\alpha$ and fiberwise action $\beta$. Then for sufficiently divisible $k$, action $\alpha$ and $\beta$ induce two commutative $\mathbb{C}^{*}$-actions on $\mathrm{H}^{0}\left(\mathcal{X}_{0}, k \mathcal{L}_{0}\right)$ (still denoted by $\alpha$ and $\beta$ ). Hence $\mathrm{H}^{0}\left(\mathcal{X}_{0}, k \mathcal{L}_{0}\right)$ becomes a $\mathbb{C}^{*} \times \mathbb{C}^{*}$-module.

In the following, we always denote the weights for $\alpha$ by $\mu \in \mathbb{Z}$ and $\nu \in \mathbb{Z}$ for $\beta$. For example, $\mathrm{H}^{0}\left(\mathcal{X}_{0}, k \mathcal{L}_{0}\right)_{\mu}$ means the weight- $\mu$ subspace w.r.t. action $\alpha$, and $\mathrm{H}^{0}\left(\mathcal{X}_{0}, k \mathcal{L}_{0}\right)_{\mu, \nu}$ means the weight $-(\mu, \nu)$ subspace w.r.t. action $\alpha \times \beta$.

In [39], the inner product of $\alpha$ and $\beta$ is defined by

$$
\begin{aligned}
\langle\alpha, \beta\rangle_{0}:= & \lim _{k \rightarrow \infty}\left[\frac{1}{k^{2} N_{k}} \sum_{\mu, \nu} \mu \nu \cdot \operatorname{dim} \mathrm{H}^{0}\left(\mathcal{X}_{0}, k \mathcal{L}_{0}\right)_{\mu, \nu}\right. \\
& \left.-\frac{1}{k^{2} N_{k}^{2}} \sum_{\mu} \mu \operatorname{dim} \mathrm{H}^{0}\left(\mathcal{X}_{0}, k \mathcal{L}_{0}\right)_{\mu} \cdot \sum_{\nu} \nu \operatorname{dim} \mathrm{H}^{0}\left(\mathcal{X}_{0}, k \mathcal{L}_{0}\right)_{\nu}\right]
\end{aligned}
$$


where $N_{k}=h^{0}(M, k L)=\frac{L^{n}}{n !} k^{n}+O\left(k^{n-1}\right)$. We use subscript " 0 " to indicate this definition using the actions on $\left(\mathcal{X}_{0}, \mathcal{L}_{0}\right)$.

We explicitly express $\langle\alpha, \beta\rangle_{0}$ by the coefficients of asymptotic expansions. First by Theorem 3.1 in [7], we know that

$$
\sum_{\mu, \nu} \mu \nu \cdot \operatorname{dim} \mathrm{H}^{0}\left(\mathcal{X}_{0}, k \mathcal{L}_{0}\right)_{\mu, \nu}=\frac{c_{0}}{(n+2) !} k^{n+2}+O\left(k^{n+1}\right)
$$

is a polynomial of $k \gg 1$ with degree at most $n+2$. Similarly, we have

$$
\sum_{\mu} \mu \operatorname{dim} \mathrm{H}^{0}\left(\mathcal{X}_{0}, k \mathcal{L}_{0}\right)_{\mu}=\frac{a_{0}}{(n+1) !} k^{n+1}+O\left(k^{n}\right) .
$$

Since the weight distribution $\mathfrak{m}_{k}(2.1)$ weakly converges to $\mathrm{DH}(\mathcal{X}, \mathcal{L}),(2.2)$ implies $a_{0}=$ $\mathcal{L}^{n+1}$.

Next consider action $\beta$. Since $\pi: \mathcal{X} \rightarrow \mathbb{P}^{1}$ is flat and $\mathcal{L}$ is relatively ample, by Corollary 12.9 [18], when $k$ is large and divisible enough, $\pi_{*}(k \mathcal{L})$ will be a vector bundle on $\mathbb{P}^{1}$ and equipped with a fiberwise action induced by $\beta$. In particular, $\mathrm{H}^{0}\left(\mathcal{X}_{0}, k \mathcal{L}_{0}\right)$ is isomorphic to $\mathrm{H}^{0}(M, k L)$ w.r.t. action $\beta$, this implies

$$
\sum_{\nu} \nu \operatorname{dim} \mathrm{H}^{0}\left(\mathcal{X}_{0}, k \mathcal{L}_{0}\right)_{\nu}=\sum_{\nu} \nu \operatorname{dim} \mathrm{H}^{0}(M, k L)_{\nu}=\frac{b_{0}}{(n+1) !} k^{n+1}+O\left(k^{n}\right) .
$$

By these expansions, we have

$$
\langle\alpha, \beta\rangle_{0}=\frac{1}{L^{n} / n !} \frac{c_{0}}{(n+2) !}-\frac{1}{\left(L^{n} / n !\right)^{2}} \frac{\mathcal{L}^{n+1}}{(n+1) !} \frac{b_{0}}{(n+1) !} .
$$

3.2. An intersection-theoretic definition for inner products . Following [7], the pullbacks of an equivariant test-configuration are regarded to be equivalent to the original one, it is natural to expect they have same inner products. Instead of checking the invariance of $\langle\alpha, \beta\rangle_{0}$, we prefer to give another definition in terms of intersection numbers, whose invariance is more apparent. Moreover, the new definition is valid for general $\mathcal{L}$. We will see that it coincides with $\langle\alpha, \beta\rangle_{0}$ when $\mathcal{L}$ is relatively ample. In the following, $(\mathcal{X}, \mathcal{L})$ is a $\mathbb{C}^{*}$-equivariant test-configuration for $(M, L)$, where $\mathcal{L}$ is not necessarily ample.

Let $\mathbb{C}^{2} \backslash\{0\} \rightarrow \mathbb{P}^{1}$ be the tautological $\mathbb{C}^{*}$-principal bundle, we consider the fiber bundle over $\mathbb{P}^{1}$ associated to action $\beta$,

$$
F: \mathcal{X}_{\beta}=\left(\mathbb{C}^{2} \backslash\{0\}\right) \times_{\beta} \mathcal{X}:=\left(\mathbb{C}^{2} \backslash\{0\}\right) \times \mathcal{X} / \sim_{\beta} \rightarrow \mathbb{P}^{1},
$$

where the equivalence relation is $(z, x) \sim_{\beta}(\tau . z, \beta(\tau) x)$, for $z \in \mathbb{C}^{2} \backslash\{0\}, x \in \mathcal{X}$ and $\tau \in \mathbb{C}^{*}$.

In the same way, $\mathcal{L}$ equipped with action $\beta$ induces a line bundle $\mathcal{L}_{\beta}$ on $\mathcal{X}_{\beta}$. By the asymptotic Riemann-Roch formula (see Theorem 1.1.24 [25]), we have expansion of Euler characteristic,

$$
\chi\left(\mathcal{X}_{\beta}, k \mathcal{L}_{\beta}\right)=\frac{\mathcal{L}_{\beta}^{n+2}}{(n+2) !} k^{n+2}+O\left(k^{n+1}\right),
$$

where $\mathcal{L}_{\beta}^{n+2}$ is intersection number on $\mathcal{X}_{\beta}$ which is of dimension $n+2$. Then our new definition is just replacing $c_{0}$ in (3.4) by the above leading coefficient.

Definition 3.1 (intersection-theoretic definition for inner products). Let $(\mathcal{X}, \mathcal{L})$ be $a \mathbb{C}^{*}$ equivariant test-configuration for $(M, L)$ with structure action $\alpha$ and fiberwise action $\beta$. 
The inner product of $\alpha$ and $\beta$ is defined by

$$
\langle\alpha, \beta\rangle=\frac{1}{L^{n} / n !} \frac{\mathcal{L}_{\beta}^{n+2}}{(n+2) !}-\frac{1}{\left(L^{n} / n !\right)^{2}} \frac{\mathcal{L}^{n+1}}{(n+1) !} \frac{b_{0}}{(n+1) !},
$$

where $b_{0}$ is given by (3.3).

In Theorem 3.9, we will show $\langle\alpha, \beta\rangle$ coincides with $\langle\alpha, \beta\rangle_{0}$ when $\mathcal{L}$ is ample. Thus it extends the original definition. By above definition, immediately we have

Proposition 3.2. Let $(\mathcal{X}, \mathcal{L})$ be a $\mathbb{C}^{*}$-equivariant test-configuration for $(M, L)$ and $g$ : $\left(\mathcal{Y}, g^{*} \mathcal{L}\right) \rightarrow(\mathcal{X}, \mathcal{L})$ be a pullback, then we have $\langle\alpha, \beta\rangle_{\mathcal{X}}=\langle\alpha, \beta\rangle_{\mathcal{Y}}$.

Proof. Denote $\mathcal{K}=g^{*} \mathcal{L}$, the fiber bundle construction induces a birational morphism $G: \mathcal{Y}_{\beta} \rightarrow \mathcal{X}_{\beta}$ such that $\mathcal{K}_{\beta}=G^{*} \mathcal{L}_{\beta}$, thus $\mathcal{K}_{\beta}^{n+2}=\mathcal{L}_{\beta}^{n+2}$. We also have $\mathcal{K}^{n+1}=\mathcal{L}^{n+1}$, and $b_{0}$ is unchanged.

3.3. Modified terms of energy functionals converge to inner products. When we consider the limit slope of modified energy functionals (e.g. modified K-energy (5.3) or Ding functional (5.2)) along a ray of metrics $\left\{\phi_{t}\right\}$, it will involve the limit of modified term $\int \dot{\phi}_{t} \theta_{X}\left(\phi_{t}\right) \mathrm{MA}\left(\phi_{t}\right)$. When the ray is induced from a test-configuration in the way given below, we show this integral converges to the inner product. In the sequel, for a testconfiguration $(\mathcal{X}, \mathcal{L})$ of $(M, L)$, we always identify $\left(\mathcal{X}_{1}, \mathcal{L}_{1}\right) \cong(M, L)$. Restricted by our method, we only consider the following two types of rays of metrics on $(\mathcal{X}, \mathcal{L})$.

Definition 3.3. (Admissible rays of metrics) Let $(\mathcal{X}, \mathcal{L})$ be a test-configuration for polarized manifold $(M, L)$. We consider a $\alpha\left(\mathbb{S}^{1}\right)$-invariant metric $\Phi$ on $\mathcal{L}$ satisfying one of the following conditions:

(A) $\Phi$ is smooth, not necessary with positive curvature (even along fibers);

(B) $\Phi$ is a locally bounded psh metric (i.e. with positive curvature current) and locally $C^{1,1}$ on $\mathcal{X} \backslash \mathcal{X}_{0}$. The most important example is the metric on $\mathcal{L}$ gives Phong-Sturm's geodesic ray [36], its $C^{1,1}$-regularity is established in [10].

Take a smooth reference metric $\psi_{0}$ on $L$. Since we identify $\left(\mathcal{X}_{1}, \mathcal{L}_{1}\right) \cong(M, L)$, the pulling-back

$$
\left\{\phi_{t}=\psi_{0}+u_{t}:=\alpha\left(e^{-\frac{t}{2}}\right)^{*} \Phi \mid t \geq 0\right\}
$$

is a ray of metrics on $L$. We call this ray of metrics is induced by $\Phi$. Let $\Omega=i \partial \bar{\partial} \Phi$ be the curvature current, we call $\left\{\phi_{t}\right\}$ is a subgeodesic ray if $\Omega$ is a positive current, and we call $\left\{\phi_{t}\right\}$ is a geodesic ray if further $\Omega^{n+1}=0$ holds.

With the assumptions in above definition, and we further assume that $\mathcal{X}$ is smooth. Let $\omega_{0}=i \partial \bar{\partial} \psi_{0}$, then the curvature form of $\phi_{t}$ is $\omega_{u_{t}}=\omega_{0}+i \partial \bar{\partial} u_{t}$, and we have

$$
\omega_{u_{t}}=\alpha\left(e^{-t / 2}\right)^{*} \Omega, \text { for } t \geq 0 .
$$

Suppose the structure $\mathbb{C}^{*}$-action $\alpha$ is generated by holomorphic vector field $W$ on $\mathcal{X}$ in the sense that

$$
\alpha\left(e^{-\frac{1}{2}(t+i s)}\right)=\exp (t \cdot \operatorname{Re} W-s \cdot \operatorname{Im} W) .
$$

Since action $\alpha$ has been lifted to $\mathcal{L}$, by (2.6), we obtain a Hamiltonian function $\Theta_{W}$ of $W$ such that

$$
\iota_{W} \Omega=i \bar{\partial} \Theta_{W}, \text { on } \mathcal{X}
$$

Taking the time derivative of

$$
\phi_{t}=\alpha\left(e^{-t / 2}\right)^{*} \Phi=\alpha\left(e^{-t_{0} / 2}\right)^{*} \alpha\left(e^{-\left(t-t_{0}\right) / 2}\right)^{*} \Phi
$$


at any $t \in \mathbb{R}$, we obtain

$$
\dot{\phi}_{t}=\dot{u}_{t}=\alpha\left(e^{-t / 2}\right)^{*} \Theta_{W}, \text { on } \mathcal{X}_{1} \cong M, \text { for } \forall t \in \mathbb{R} .
$$

Next we assume $(\mathcal{X}, \mathcal{L})$ is a $\mathbb{C}^{*}$-equivariant test-configuration with fiberwise action $\beta$, and require $\Phi$ is $\beta\left(\mathbb{S}^{1}\right)$-invariant. Suppose the action $\beta$ is generated by holomorphic vector field $X$ on $\mathcal{X}$ in the sense that

$$
\beta\left(e^{-\frac{1}{2}(t+i s)}\right)=\exp (t \cdot \operatorname{Re} X-s \cdot \operatorname{Im} X) .
$$

Note that $X$ is tangent to each fiber except $\mathcal{X}_{0}$. In the same way, we obtain a Hamiltonian function $\Theta_{X}$ for $X$ from the lifted action $\beta$, it satisfies

$$
\iota_{X} \Omega=i \bar{\partial} \Theta_{X} \text {, on } \mathcal{X} \text {. }
$$

Lemma 3.4. Let $f$ be any $C^{1}$ function on $\mathbb{R}$, then the integral $\int_{\mathcal{X}_{\tau}} f\left(\Theta_{X}\right) \Omega^{n}$ is independent of $\tau \in \mathbb{P}^{1} \backslash\{0\}$.

Proof. Let $I(\tau)=\int_{\mathcal{X}_{\tau}} f\left(\Theta_{X}\right) \Omega^{n}$, for $\tau \in \mathbb{P}^{1} \backslash\{0\}$. By the property of fiber integration (over $\mathbb{P}^{1} \backslash\{0\}$ ) and (3.11), we have

$$
i \bar{\partial} I(\tau)=\int_{\mathcal{X} / \mathbb{P}^{1}} f^{\prime}\left(\Theta_{X}\right) \iota_{X} \Omega \wedge \Omega^{n}=\frac{1}{n+1} \int_{\mathcal{X} / \mathbb{P}^{1}} f^{\prime}\left(\Theta_{X}\right) \iota_{X}\left(\Omega^{n+1}\right) .
$$

Since $X$ is tangent to each fiber, the last integral vanishes. Consider that $I(\tau)$ is real-valued, thus it must be a constant.

Let

$$
\tilde{\Theta}_{X}=\Theta_{X}-\frac{1}{V} \int_{\mathcal{X}_{1}} \Theta_{X} \Omega^{n},
$$

then above lemma implies $\int_{\mathcal{X}_{\tau}} \tilde{\Theta}_{X} \Omega^{n}=0$ for each $\tau \in \mathbb{C}^{*}$. We define

$$
\theta_{X}\left(u_{t}\right):=\alpha\left(e^{-t / 2}\right)^{*} \tilde{\Theta}_{X}, \text { on } \mathcal{X}_{1} \simeq M,
$$

then $\int_{M} \theta_{X}\left(u_{t}\right) \omega_{u_{t}}^{n}=0$, and (3.11) is translated into $\iota_{X} \omega_{u_{t}}=i \bar{\partial} \theta_{X}\left(u_{t}\right)$. Thus $\theta_{X}\left(u_{t}\right)$ is the normalized Hamiltonian function for $X$ w.r.t. $\omega_{u_{t}}$.

Proposition 3.5. With the assumptions and notations as above. We assume $\mathcal{X}$ is smooth and $\Phi$ is a metric on $\mathcal{L}$ satisfying condition $(A)$ or $(B)$ in Definition 3.3. Then we have

$$
\lim _{t \rightarrow+\infty} \frac{1}{V} \int_{M} \dot{u}_{t} \theta_{X}\left(u_{t}\right) \omega_{u_{t}}^{n}=\frac{1}{2 \pi(n+1) V} \int_{\mathcal{X}} \tilde{\Theta}_{X} \Omega^{n+1}
$$

Proof. First we suppose $\Phi$ satisfies condition (A). We define a function on $\mathbb{P}^{1} \backslash\{0\}$,

$$
F(\tau):=\int_{\mathcal{X}_{\tau}} \Theta_{W} \cdot \tilde{\Theta}_{X} \Omega^{n} .
$$

By the assumptions, $\Theta_{W}$ and $\Omega$ are $\alpha\left(\mathbb{S}^{1}\right)$-invariant, $\alpha$ and $\beta$ commutes with each other, thus $\tilde{\Theta}_{X}$ is also $\alpha\left(\mathbb{S}^{1}\right)$-invariant. This follows that $F(\tau)$ only depends on $|\tau|$. By relations (3.9) and (3.13), we have

$$
F\left(e^{-t / 2}\right)=\int_{M} \dot{u}_{t} \theta_{X}\left(u_{t}\right) \omega_{u_{t}}^{n}, \text { for } t \in \mathbb{R} .
$$

Since action $\alpha$ on $(\mathcal{X}, \mathcal{L})_{\infty}$ is trivial, thus $\left.\Theta_{W}\right|_{\mathcal{X}_{\infty}} \equiv 0$, so $F(\infty)=0$.

In terms of fiber integration along $\pi:\left.\mathcal{X}\right|_{\mathbb{P}^{1} \backslash\{0\}} \rightarrow \mathbb{P}^{1} \backslash\{0\}$, we have

$$
F=\int_{\mathcal{X} /(\mathbb{P} \backslash \backslash 0)} \Theta_{W} \cdot \tilde{\Theta}_{X} \Omega^{n}
$$


Next we show

$$
i \bar{\partial}\left(F(\tau) \frac{d \tau}{\tau}\right)=\frac{1}{n+1} \int_{\mathcal{X} / \mathbb{C}^{*}}\left(\tilde{\Theta}_{X} \Omega^{n+1}\right), \text { on } \mathbb{C}^{*},
$$

where the RHS is fiber integration over $\mathbb{C}^{*}$. Exchange $\bar{\partial}$-operator with fiber integration, and use relations (3.8) and (3.11), we have

$$
\begin{aligned}
i \bar{\partial} F(\tau) \wedge d \tau & =\left(\int_{\mathcal{X} / \mathbb{C}^{*}} \iota_{W} \Omega \wedge \tilde{\Theta}_{X} \Omega^{n}+\int_{\mathcal{X} / \mathbb{C}^{*}} \Theta_{W} \cdot \iota_{X} \Omega \wedge \Omega^{n}\right) \wedge d \tau \\
& =\frac{1}{n+1} \int_{\mathcal{X} / \mathbb{C}^{*}} \tilde{\Theta}_{X} \iota_{W}\left(\Omega^{n+1}\right) \wedge \pi^{*}(d \tau) \\
& =\frac{\tau}{n+1} \int_{\mathcal{X} / \mathbb{C}^{*}} \tilde{\Theta}_{X} \Omega^{n+1} .
\end{aligned}
$$

The second integral in the first row vanishes since $X$ is tangent to each fiber. The second row is by projection formula. The third row uses fact $\pi_{*}(W)=-\tau \frac{\partial}{\partial \tau}$.

Let $A_{r, R}:=\{\tau|r \leq| \tau \mid \leq R\}$, applying Stokes formula on $A_{r, R}$, we have

$$
\int_{A_{r, R}} i \bar{\partial}\left(F(\tau) \frac{d \tau}{\tau}\right)=i \int_{A_{r, R}} d\left(F(\tau) \frac{d \tau}{\tau}\right)=2 \pi(F(r)-F(R)) .
$$

Let $r \rightarrow 0$ and $R \rightarrow+\infty$, then (3.14) follows from (3.15).

When $\Phi$ satisfies condition (B), then $\Phi$ has $C^{1,1}$-regularity on $\mathcal{X} \backslash \mathcal{X}_{0}$. To ensure the above arguments still work, we note: (1) the fiber integration is taken over $\mathbb{P}^{1} \backslash\{0\} ; \Omega$ is a 2 -form with $L^{\infty}$-coefficients on $\mathcal{X} \backslash \mathcal{X}_{0}$; the associated functions $\Theta_{W}, \tilde{\Theta}_{X}$ (locally is $X \Phi$ ) are $C^{0,1}$ on $\mathcal{X} \backslash \mathcal{X}_{0}$; (2) Over the total space $\mathcal{X}, \Omega^{n+1}$ is interpreted as the nonpluripolar product. So the above arguments still work.

Next we use the equivariant HRR formula to connect the RHS integral of (3.14) to the equivariant Euler characteristic.

Definition 3.6. Let $(\mathcal{X}, \mathcal{L})$ be a $\mathbb{C}^{*}$-equivariant test-configuration for $(M, L)$ with fiberwise action $\beta$, the degree-1 equivariant Euler characteristic is defined by

$$
\chi_{1}^{\beta}(\mathcal{X}, k \mathcal{L}):=\sum_{q=0}^{n+1}(-1)^{q} \sum_{\nu \in \mathbb{Z}} \nu \cdot \operatorname{dim} H^{q}(\mathcal{X}, k \mathcal{L})_{\nu},
$$

where $H^{q}(\mathcal{X}, k \mathcal{L})_{\nu}$ is the weight- $\nu$ subspace of $H^{q}(\mathcal{X}, k \mathcal{L})$ with respect to $\mathbb{C}^{*}$-action $\beta$.

Theorem 3.7. (1) (modified terms converge to inner products) Let $(\mathcal{X}, \mathcal{L})$ be a $\mathbb{C}^{*}$-equivariant test-configuration for $(M, L)$ with structure action $\alpha$ and fiberwise action $\beta$ generated by $X$. $\mathcal{X}$ is not necessarily smooth. Let $\Phi$ be a $\alpha\left(\mathbb{S}^{1}\right) \times \beta\left(\mathbb{S}^{1}\right)$-invariant metric on $\mathcal{L}$ satisfying condition $(A)$ or $(B)$ in Definition 3.3. It induces a ray of metrics $\left\{\phi_{t}=\psi_{0}+u_{t}\right\}_{t \geq 0}$ on $L$ with curvature form $\omega_{u_{t}}$. Let $\theta_{X}\left(u_{t}\right)$ be the normalized Hamiltonian function of $X$ w.r.t. $\omega_{u_{t}}$. Then we have

$$
\lim _{t \rightarrow+\infty} \frac{1}{V} \int_{M} \dot{u}_{t} \theta_{X}\left(u_{t}\right) \omega_{u_{t}}^{n}=\langle\alpha, \beta\rangle .
$$

(2) (Integral formula for inner products) Let $(\mathcal{X}, \mathcal{L})$ be a $\mathbb{C}^{*}$-equivariant test-configuration for $(M, L)$. Assume $\mathcal{X}$ is smooth, given a smooth 2-form $\Omega \in 2 \pi c_{1}(\mathcal{L})$ and function $\Theta$ on 
$\mathcal{X}$ satisfying $\iota_{X} \Omega=i \bar{\partial} \Theta$ and $\int_{\mathcal{X}_{1}} \Theta \Omega^{n}=0$, then we have

$$
\langle\alpha, \beta\rangle=\frac{1}{(n+1) L^{n}} \int_{\mathcal{X}} \Theta\left(\frac{\Omega}{2 \pi}\right)^{n+1} .
$$

Proof. If the total space $\mathcal{X}$ is singular, we take a $\mathbb{C}^{*} \times \mathbb{C}^{*}$-equivariant resolution $p: \mathcal{X}^{\prime} \rightarrow \mathcal{X}$ which is equivariant and an isomorphism on $p^{-1}\left(\mathcal{X} \backslash \mathcal{X}_{0}\right)$. Let $\mathcal{L}^{\prime}=p^{*} \mathcal{L}$, then $\left(\mathcal{X}^{\prime}, \mathcal{L}^{\prime}\right)$ has same inner product $\langle\alpha, \beta\rangle$ with $(\mathcal{X}, \mathcal{L})$ by Proposition 3.2. Endowing $\mathcal{L}^{\prime}$ with the pullingback metric $p^{*} \Phi$, it induces the same ray of metrics on $L$, so gives the same limit on the LHS of (3.16). So if we establish (3.16) for $\left(\mathcal{X}^{\prime}, \mathcal{L}^{\prime}\right)$, then it also holds for $(\mathcal{X}, \mathcal{L})$. In the following we can assume $\mathcal{X}$ is smooth.

Since $\Omega=i \partial \bar{\partial} \Phi \in 2 \pi c_{1}(\mathcal{L}), V=(2 \pi)^{n} L^{n}$, by Proposition 3.5 and (3.12), the limit on the LHS of (3.16) is equal to

$$
\frac{1}{L^{n} / n !} \int_{\mathcal{X}} \Theta_{X} \frac{(\Omega / 2 \pi)^{n+1}}{(n+1) !}-\frac{1}{\left(L^{n} / n !\right)^{2}} \frac{\mathcal{L}^{n+1}}{(n+1) !} \cdot \int_{\mathcal{X}_{1}} \Theta_{X} \frac{(\Omega / 2 \pi)^{n}}{n !} .
$$

Now we use equivariant HRR formula to relate these integrals to the equivariant Euler characteristic.

For the integral over $\mathcal{X}_{1}$, apply (2.7) to datum $\left(M, L,\left.\Phi\right|_{\mathcal{X}_{1}}, \beta\right)$, it gives

$$
\sum_{\nu \in \mathbb{Z}} \nu \operatorname{dim} \mathrm{H}^{0}(M, k L)_{\nu}=\int_{\mathcal{X}_{1}} \Theta_{X} \frac{(\Omega / 2 \pi)^{n}}{n !} \cdot k^{n+1}+O\left(k^{n}\right), \text { for } k \gg 1 .
$$

For the integral over $\mathcal{X}$, apply (2.7) to datum $(\mathcal{X}, \mathcal{L}, \Phi, \beta)$, it gives

$$
\chi_{1}^{\beta}(\mathcal{X}, k \mathcal{L})=\int_{\mathcal{X}} \Theta_{X} \frac{(\Omega / 2 \pi)^{n+1}}{(n+1) !} \cdot k^{n+2}+O\left(k^{n+1}\right), \text { for } k \gg 1 .
$$

Combing these two expansions with the below lemma, which connects $\chi_{1}^{\beta}(\mathcal{X}, k \mathcal{L})$ to $\chi\left(\mathcal{X}_{\beta}, k \mathcal{L}_{\beta}\right)$, we have

$$
\mathcal{L}_{\beta}^{n+2}=(n+2) ! \cdot \int_{\mathcal{X}} \Theta_{X} \frac{(\Omega / 2 \pi)^{n+1}}{(n+1) !} .
$$

Then (3.16) follows from our Definition (3.6) of inner product.

For the part (2), choose a metric on $\mathcal{L}$ with curvature form $\Omega$, then we obtain a Hamiltonian function $\Theta_{X}$ from the lifted action $\beta$. Its normalization $\tilde{\Theta}_{X}$ by (3.12) must coincide with $\Theta$, then (3.17) follows in the same way as above.

Lemma 3.8. Let $(\mathcal{X}, \mathcal{L})$ be a $\mathbb{C}^{*}$-equivariant test-configuration for $(M, L)$ with fiberwise action $\beta$. $\left(\mathcal{X}_{\beta}, \mathcal{L}_{\beta}\right)$ is the fiber bundle (3.5) associated to $\beta$. Then we have relation

$$
\chi\left(\mathcal{X}_{\beta}, k \mathcal{L}_{\beta}\right)=\chi_{1}^{\beta}(\mathcal{X}, k \mathcal{L})+\chi(\mathcal{X}, k \mathcal{L}) .
$$

Thus $\chi_{1}^{\beta}(\mathcal{X}, k \mathcal{L})$ is a polynomial of $k$ with degree at most $n+2$ when $k \gg 1 . \chi\left(\mathcal{X}_{\beta}, k \mathcal{L}_{\beta}\right)$ and $\chi_{1}^{\beta}(\mathcal{X}, k \mathcal{L})$ have same coefficient of the leading term $k^{n+2}$.

Proof. Consider the Leray's spectral sequence associated to $F: \mathcal{X}_{\beta} \rightarrow \mathbb{P}^{1}$ (see [17] Section 4.6.3 for details),

$$
E_{2}^{p, q}=\mathrm{H}^{p}\left(\mathbb{P}^{1}, \mathrm{R}^{q} F_{*}\left(k \mathcal{L}_{\beta}\right)\right) \Rightarrow E^{p+q}=\mathrm{H}^{p+q}\left(\mathcal{X}_{\beta}, k \mathcal{L}_{\beta}\right) .
$$

Since the Euler characteristic $\sum_{p, q}(-1)^{p+q} \operatorname{dim} E_{r}^{p, q}$ of each page are same with each other, we have

$$
\chi\left(\mathcal{X}_{\beta}, k \mathcal{L}_{\beta}\right)=\sum_{q}(-1)^{q} \chi\left(\mathbb{P}^{1}, \mathbf{R}^{q} F_{*}\left(k \mathcal{L}_{\beta}\right)\right) .
$$


We claim that

$$
\mathrm{R}^{q} F_{*}\left(k \mathcal{L}_{\beta}\right)=\bigoplus_{\nu \in \mathbb{Z}} \mathrm{H}^{q}(\mathcal{X}, k \mathcal{L})_{\nu} \otimes \mathcal{O}_{\mathbb{P}^{1}}(\nu) .
$$

Decomposing $\mathrm{H}^{q}(\mathcal{X}, k \mathcal{L})$ with respect to action $\beta$, we have

$$
\begin{aligned}
\mathrm{R}^{q} F_{*}\left(k \mathcal{L}_{\beta}\right) & =\left(\mathbb{C}^{2} \backslash\{0\}\right) \times{ }_{\beta} \mathrm{H}^{q}(\mathcal{X}, k \mathcal{L}) \\
& =\bigoplus_{\nu \in \mathbb{Z}}\left(\mathbb{C}^{2} \backslash\{0\}\right) \times_{\tau^{\nu}} \mathrm{H}^{q}(\mathcal{X}, k \mathcal{L})_{\nu},
\end{aligned}
$$

where the $\mathbb{C}^{*}$-action on $\mathrm{H}^{q}(\mathcal{X}, k \mathcal{L})_{\nu}$ is multiplication by character $\tau^{\nu}$. Since $\left(\mathbb{C}^{2} \backslash\{0\}\right) \times \tau^{\nu}$ $\mathbb{C}=\mathcal{O}_{\mathbb{P}^{1}}(\nu),(3.18)$ follows.

Now taking the Euler characteristic of both sides of (3.18), using $\chi\left(\mathbb{P}^{1}, \mathcal{O}_{\mathbb{P}^{1}}(\nu)\right)=\nu+1$, we have

$$
\begin{aligned}
\chi\left(\mathcal{X}_{\beta}, k \mathcal{L}_{\beta}\right) & =\sum_{q}(-1)^{q} \sum_{\nu}(\nu+1) \operatorname{dim} \mathrm{H}^{q}(\mathcal{X}, k \mathcal{L})_{\nu} \\
& =\chi_{1}^{\beta}(\mathcal{X}, k \mathcal{L})+\chi(\mathcal{X}, k \mathcal{L}) .
\end{aligned}
$$

The last statement follows by $\chi(\mathcal{X}, k \mathcal{L})$ is a polynomial of $k$ with degree $\leq n+1$.

\subsection{Coincidence of two definitions for inner products.}

Theorem 3.9. Let $(\mathcal{X}, \mathcal{L})$ be a $\mathbb{C}^{*}$-equivariant test-configuration for $(M, L)$. If $\mathcal{L}$ is relatively ample, then $\langle\alpha, \beta\rangle$ defined by (3.6) is same to $\langle\alpha, \beta\rangle_{0}$ defined by (3.4).

Proof. We only need to show $c_{0}$ in expansion (3.2) is equal to $\mathcal{L}_{\beta}^{n+2}$. By Lemma 3.8, this is same to show $c_{0}$ is the leading coefficient of $\chi_{1}^{\beta}(\mathcal{X}, k \mathcal{L})$.

Consider the Leray's spectral sequence associated to $\pi: \mathcal{X} \rightarrow \mathbb{P}^{1}$,

$$
E_{2}^{p, q}=\mathrm{H}^{p}\left(\mathbb{P}^{1}, \mathrm{R}^{q} \pi_{*}(k \mathcal{L})\right) \Rightarrow E^{p+q}=\mathrm{H}^{p+q}(\mathcal{X}, k \mathcal{L}) .
$$

Without loss of the information of actions, the spectral sequence is taking in the abelian category of $\mathbb{C}^{*} \times \mathbb{C}^{*}$-modules, namely each term $E_{r}^{p, q}$ is equipped with a $\mathbb{C}^{*} \times \mathbb{C}^{*}$-action induced by $\alpha \times \beta$ and the differentials $d_{r}^{p, q}$ are equivariant w.r.t. these actions.

Since the differentials on page- 2 are $d_{2}^{p, q}: E_{2}^{p, q} \rightarrow E_{2}^{p+2, q-1}$ and $E_{2}^{p, q}=0$ when $p \notin\{0,1\}$ (since $\operatorname{dim} \mathbb{P}^{1}=1$ ), above spectral sequence actually degenerates at page- 2 . Combining this with the fact that abelian category of $\mathbb{C}^{*} \times \mathbb{C}^{*}$-modules is semi-simple (i.e. every short exact sequence splits), we obtain

$$
\begin{aligned}
& \mathrm{H}^{q}(\mathcal{X}, k \mathcal{L}) \cong \mathrm{H}^{0}\left(\mathbb{P}^{1}, \mathrm{R}^{q} \pi_{*}(k \mathcal{L})\right) \bigoplus \mathrm{H}^{1}\left(\mathbb{P}^{1}, \mathrm{R}^{q-1} \pi_{*}(k \mathcal{L})\right), \text { for } q \geq 1 ; \\
& \mathrm{H}^{0}(\mathcal{X}, k \mathcal{L}) \cong \mathrm{H}^{0}\left(\mathbb{P}^{1}, \pi_{*}(k \mathcal{L})\right),
\end{aligned}
$$

all these isomorphisms are between $\mathbb{C}^{*} \times \mathbb{C}^{*}$-modules.

Now we use the assumption of ampleness. When $k$ is sufficiently large and divisible, we have $\mathrm{R}^{q} \pi_{*}(k \mathcal{L})=0$ for any $q>0$. Then above isomorphisms imply

$$
\mathrm{H}^{q}(\mathcal{X}, k \mathcal{L}) \cong \mathrm{H}^{q}\left(\mathbb{P}^{1}, \pi_{*}(k \mathcal{L})\right), \text { for } q=0,1 ; \mathrm{H}^{q}(\mathcal{X}, k \mathcal{L})=0, \text { for } q>1
$$

as $\mathbb{C}^{*} \times \mathbb{C}^{*}$-modules when $k \gg 1$.

Let $\mathcal{E}:=\pi_{*}(k \mathcal{L})$ be the direct image, since $\pi$ is flat, by [18] Corollary 12.9, when $k \gg 1$, $\mathcal{E}$ is a vector bundle over $\mathbb{P}^{1}$ with rank $N_{k}$. Moreover, $\mathcal{E}$ is equipped with two commutative $\mathbb{C}^{*}$-actions, still denoted by $\alpha$ and $\beta$. By action $\beta, \mathcal{E}$ is decomposed to subbundles,

$$
\mathcal{E}=\bigoplus_{\nu \in \mathbb{Z}} \mathcal{E}_{\nu}
$$


where for each point $\tau \in \mathbb{P}^{1},\left.\mathcal{E}_{\nu}\right|_{\tau}$ is the weight- $\nu$ subspace of $\mathrm{H}^{0}\left(\mathcal{X}_{\tau}, k \mathcal{L}_{\tau}\right)$. On the other hand, since $\alpha$ commutes with $\beta$, thus $\alpha$ preserves each bundle $\mathcal{E}_{\nu}$.

Direct sum $\mathrm{H}^{p}\left(\mathbb{P}^{1}, \mathcal{E}\right)=\bigoplus_{\nu} \mathrm{H}^{p}\left(\mathbb{P}^{1}, \mathcal{E}_{\nu}\right)$ can be seen as the weight decomposition w.r.t. $\beta$, then isomorphism (3.19) tells us $\mathrm{H}^{p}(\mathcal{X}, k \mathcal{L})_{\nu}=\mathrm{H}^{p}\left(\mathbb{P}^{1}, \mathcal{E}_{\nu}\right)$. This follows that

$$
\chi_{1}^{\beta}(\mathcal{X}, k \mathcal{L})=\sum_{p}(-1)^{p} \sum_{\nu} \nu \cdot \operatorname{dim} \mathrm{H}^{p}\left(\mathbb{P}^{1}, \mathcal{E}_{\nu}\right)=\sum_{\nu} \nu \cdot \chi\left(\mathbb{P}^{1}, \mathcal{E}_{\nu}\right) .
$$

Next we consider each subbundle $\mathcal{E}_{\nu} \rightarrow \mathbb{P}^{1}$ equipped with a $\mathbb{C}^{*}$-action $\alpha$. Note the action on $\left.\mathcal{E}_{\nu}\right|_{\infty}$ is trivial since the compactification we made. Using the following lemma, take the vector bundle $E$ therein to be $\mathcal{E}_{\nu}$. Since $\left.\mathcal{E}_{\nu}\right|_{0}=\mathrm{H}^{0}\left(\mathcal{X}_{0}, k \mathcal{L}_{0}\right)_{\nu}$, it yields

$$
\chi\left(\mathbb{P}^{1}, \mathcal{E}_{\nu}\right)=\sum_{\mu} \mu \cdot \operatorname{dim} \mathrm{H}^{0}\left(\mathcal{X}_{0}, k \mathcal{L}_{0}\right)_{\mu, \nu}+\operatorname{rank} \mathcal{E}_{\nu} .
$$

Put this into (3.20), we obtain

$$
\chi_{1}^{\beta}(\mathcal{X}, k \mathcal{L})=\sum_{\mu, \nu} \mu \nu \cdot \operatorname{dim} \mathrm{H}^{0}\left(\mathcal{X}_{0}, k \mathcal{L}_{0}\right)_{\mu, \nu}+\sum_{\nu} \nu \cdot \operatorname{dim} \mathrm{H}^{0}(M, k L)_{\nu} .
$$

Since the second term on the RHS has lower degree than others, the leading coefficient of $\chi_{1}^{\beta}(\mathcal{X}, k \mathcal{L})$ is $c_{0}$, defined by (3.2).

Lemma 3.10. Let $E$ be a rank $r$ holomorphic vector bundle over $\mathbb{P}^{1}=\mathbb{C} \cup\{\infty\}$. There is $a \mathbb{C}^{*}$-action on $E$ covers the multiplication action on $\mathbb{P}^{1}$. Then

$$
\chi\left(\mathbb{P}^{1}, E\right)=r+w_{0}-w_{\infty},
$$

where $w_{0}$ and $w_{\infty}$ is the total weight of action on $E_{0}$ and $E_{\infty}$ respectively.

Proof. The Hirzebruch-Riemann-Roch theorem says

$$
\chi\left(\mathbb{P}^{1}, E\right)=\int_{\mathbb{P}^{1}} \operatorname{ch}(E) \cdot \operatorname{td}\left(T_{\mathbb{P}^{1}}\right) .
$$

Since the Chern character $\operatorname{ch}(E)=r+c_{1}(E)$, and the Todd class $\operatorname{td}\left(T_{\mathbb{P}^{1}}\right)=1+\frac{1}{2} c_{1}\left(T_{\mathbb{P}^{1}}\right)=$ $1+c_{1}(\mathcal{O}(1))$. Thus

$$
\chi\left(\mathbb{P}^{1}, E\right)=r+\int_{\mathbb{P}^{1}} c_{1}(E)=r+\operatorname{deg}\left(\wedge^{r} E\right) .
$$

Denote the line bundle $\wedge^{r} E$ by $K$. Take any nonzero $s_{1} \in K_{1}$, define a meromorphic section of $K$ by $s(\tau)=\tau . s_{1}$, for $\tau \in \mathbb{C}^{*}$. It is easy to see that the divisor defined by $s$ is $w_{0} \cdot 0-w_{\infty} \cdot \infty$, where $w_{0}$ and $w_{\infty}$ are weights of $\mathbb{C}^{*}$-action on $K_{0}$ and $K_{\infty}$ respectively, which are also the total weights of $\mathbb{C}^{*}$-action on $E_{0}$ and $E_{\infty}$. The degree of $K$ is $w_{0}-$ $w_{\infty}$

\section{From Monge-Ampère measures to Duistermaat-Heckman measures}

Let $(\mathcal{X}, \mathcal{L})$ be a test-configuration for $(M, L)$ with a metric $\Phi$ on $\mathcal{L}$, and $\left\{\phi_{t}\right\}$ be the induced ray of metrics on $L$. Suppose the curvature forms $\omega_{u_{t}}=i \partial \bar{\partial} \phi_{t}$ are nonnegative. Then the pushforwards of $\frac{1}{V} \omega_{u_{t}}^{n}$ by function $\dot{u}_{t}$ are probability measures on $\mathbb{R}$. When $\left\{\phi_{t}\right\}$ is Phong-Sturm's geodesic ray, Hisamoto [20] showed them weakly converge to $\operatorname{DH}(\mathcal{X}, \mathcal{L})$. In this section, we extend this result to some general rays, such as the ray induced by a smooth metric $\Phi$ with no curvature restriction. The proof runs along a similar route as Theorem 3.7, hence is different from [20]. 
4.1. From the limit of integrals to the equivariant Euler characteristic. In this subsection, $(\mathcal{X}, \mathcal{L})$ is not necessarily ample. The following proposition convert the limit into an integral over the total space.

Proposition 4.1. Let $(\mathcal{X}, \mathcal{L})$ be a test-configuration for $(M, L)$ with smooth $\mathcal{X}$. Let $\Phi$ be a $\alpha\left(\mathbb{S}^{1}\right)$-invariant metric on $\mathcal{L}$ satisfying condition $(A)$ or $(B)$ in Definition 3.3. It induces a ray of metrics $\left\{\phi_{t}=\psi_{0}+u_{t}\right\}_{t \geq 0}$ on L. Let $\Omega=i \partial \bar{\partial} \Phi$ be the curvature current, and $\Theta_{W}$ be the Hamiltonian function of vector field $W$ induced by the lifted action $\alpha$. Let $f$ be any $C^{1}$ function, then we have

$$
\lim _{t \rightarrow \infty} \frac{1}{V} \int_{M} f\left(\dot{u}_{t}\right) \omega_{u_{t}}^{n}=\frac{1}{L^{n} / n !} \int_{\mathcal{X}} f^{\prime}\left(\Theta_{W}\right) \frac{(\Omega / 2 \pi)^{n+1}}{(n+1) !}+f(0) .
$$

Proof. The proof is similar with Proposition 3.5. We define $F(\tau):=\int_{\mathcal{X}_{\tau}} f\left(\Theta_{W}\right) \Omega^{n}$ for $\tau \in \mathbb{P}^{1} \backslash\{0\}$, it only depends on $|\tau|$. We know $F\left(e^{-t / 2}\right)=\int_{M} f\left(\dot{u}_{t}\right) \omega_{u_{t}}^{n}, F(\infty)=f(0) V$. A direct computation shows that

$$
i \bar{\partial}\left(F(\tau) \frac{d \tau}{\tau}\right)=\frac{1}{n+1} \pi_{*}\left(f^{\prime}\left(\Theta_{W}\right) \Omega^{n+1}\right), \text { on } \mathbb{C}^{*} .
$$

Let $A_{r, R}:=\{\tau|r \leq| \tau \mid \leq R\}$, by Stokes' formula, we have

$$
\int_{A_{r, R}} i \bar{\partial}\left(F(\tau) \frac{d \tau}{\tau}\right)=i \int_{A_{r, R}} d\left(F(\tau) \frac{d \tau}{\tau}\right)=2 \pi(F(r)-F(R)),
$$

then let $r \rightarrow 0$ and $R \rightarrow+\infty$, (4.1) follows.

We will take $f=x^{d+1}$ and apply equivariant HRR formula to relate the above integral over $\mathcal{X}$ to the equivariant Euler characteristic.

Definition 4.2. For a test-configuration $(\mathcal{X}, \mathcal{L})$, the degree-d equivariant Euler characteristic is defined by

$$
\chi_{d}^{\alpha}(\mathcal{X}, k \mathcal{L}):=\sum_{q=0}^{n+1}(-1)^{q} \sum_{\mu \in \mathbb{Z}} \frac{\mu^{d}}{d !} \cdot \operatorname{dim} H^{q}(\mathcal{X}, k \mathcal{L})_{\mu},
$$

where $H^{q}(\mathcal{X}, k \mathcal{L})_{\mu}$ is the weight- $\mu$ subspace w.r.t. structure action $\alpha$. Note that we use different action comparing with $\chi_{1}^{\beta}$.

Since we only apply HRR formula on smooth $\mathcal{X}$, same with the proof of Theorem 3.7, we pass to the resolution and then come back. For this, we need the following invariance of the leading term of $\chi_{d}^{\alpha}(\mathcal{X}, k \mathcal{L})$.

Proposition 4.3. When $k \gg 1, \chi_{d}^{\alpha}(\mathcal{X}, k \mathcal{L})$ is a polynomial of $k$ with degree at most $n+$ $1+d$. If $\left(\mathcal{X}^{\prime}, \mathcal{L}^{\prime}\right)$ is a pullback of $(\mathcal{X}, \mathcal{L})$, the coefficient of term $k^{n+1+d}$ of $\chi_{d}^{\alpha}(\mathcal{X}, k \mathcal{L})$ is same with that of $\chi_{d}^{\alpha}\left(\mathcal{X}^{\prime}, k \mathcal{L}^{\prime}\right)$.

Proof. The proof is similar with Lemma 3.8. We express the leading coefficient by intersection number.

Similar to the bundle construction (3.5), we take the tautological $\mathbb{C}^{*}$-principal bundle $\mathbb{C}^{d+1} \backslash\{0\} \rightarrow \mathbb{P}^{d}$, define fiber bundle

$$
F: \mathcal{X}_{\alpha, d}:=\left(\mathbb{C}^{d+1} \backslash\{0\}\right) \times_{\alpha} \mathcal{X} \rightarrow \mathbb{P}^{d}
$$

and line bundle $\mathcal{L}_{\alpha, d}$ on $\mathcal{X}_{\alpha, d}$ in the similar way. 
Then consider the Leray spectral sequence associated to $F$, the invariance of Euler characteristic for each page gives us

$$
\chi\left(\mathcal{X}_{\alpha, d}, k \mathcal{L}_{\alpha, d}\right)=\sum_{q}(-1)^{q} \chi\left(\mathbb{P}^{d}, \mathrm{R}^{q} F_{*}\left(k \mathcal{L}_{\alpha, d}\right)\right)
$$

Similar with (3.18), we can show

$$
\mathrm{R}^{q} F_{*}\left(k \mathcal{L}_{\alpha, d}\right)=\bigoplus_{\mu \in \mathbb{Z}} \mathrm{H}^{q}(\mathcal{X}, k \mathcal{L})_{\mu} \otimes \mathcal{O}_{\mathbb{P}^{d}}(\mu)
$$

It follows that

$$
\chi\left(\mathcal{X}_{\alpha, d}, k \mathcal{L}_{\alpha, d}\right)=\sum_{\mu} \sum_{q}(-1)^{q} \chi\left(\mathbb{P}^{d}, \mathcal{O}_{\mathbb{P}^{d}}(\mu)\right) \cdot \operatorname{dim} \mathrm{H}^{q}(\mathcal{X}, k \mathcal{L})_{\mu} .
$$

Since $\chi\left(\mathbb{P}^{d}, \mathcal{O}_{\mathbb{P}^{d}}(\mu)\right)=\frac{\mu^{d}}{d !}+O\left(\mu^{d-1}\right)$ is a polynomial of $\mu$, and $\chi\left(\mathcal{X}_{\alpha, d}, k \mathcal{L}_{\alpha, d}\right)$ is a polynomial of $k$ with degree $\leq n+1+d$, by induction on $d$, we can show that $\chi_{d}^{\alpha}(\mathcal{X}, k \mathcal{L})$ is a polynomial of $k$ with degree $\leq n+1+d$ when $k \gg 1$. Moreover, by above identity, we see the coefficient of term $k^{n+1+d}$ of $\chi_{d}^{\alpha}(\mathcal{X}, k \mathcal{L})$ is $\mathcal{L}_{\alpha, d}^{n+1+d} /(n+1+d)$ !, then the invariance of leading coefficients under pullback follows.

Now we connect the limit with the equivariant Euler characteristic via equivariant HRR formula.

Proposition 4.4. With same assumptions as Proposition 4.1, except that $\mathcal{X}$ might be singular. For any integer $d \geq 0$, we have

$$
\lim _{t \rightarrow \infty} \frac{1}{V} \int_{M} \frac{\left(\dot{u}_{t}\right)^{d+1}}{(d+1) !} \omega_{u_{t}}^{n}=\lim _{k \rightarrow \infty} \frac{\chi_{d}^{\alpha}(\mathcal{X}, k \mathcal{L})}{k^{d+1} N_{k}} .
$$

Proof. If $\mathcal{X}$ is singular, then take an equivariant resolution $p: \mathcal{X}^{\prime} \rightarrow \mathcal{X}$. Let $\mathcal{L}^{\prime}=p^{*} \mathcal{L}$, then $\left(\mathcal{X}^{\prime}, \mathcal{L}^{\prime}\right)$ is a pullback of $(\mathcal{X}, \mathcal{L})$. Endowing $\mathcal{L}^{\prime}$ with pullback metric $p^{*} \Phi$, then the LHS limits of (4.2) are same for $\mathcal{X}^{\prime}$ and $\mathcal{X}$. On the other hand, by Proposition 4.3, the RHS leading coefficients are also same for $\mathcal{X}^{\prime}$ and $\mathcal{X}$. Hence it is sufficient to consider the case of smooth $\mathcal{X}$.

Take $f(x)=x^{d+1} /(d+1)$ ! in Proposition 4.1 , it yields

$$
\lim _{t \rightarrow \infty} \frac{1}{V} \int_{M} \frac{\left(\dot{u}_{t}\right)^{d+1}}{(d+1) !} \omega_{u_{t}}^{n}=\frac{1}{L^{n} / n !} \int_{\mathcal{X}} \frac{\Theta_{W}^{d}}{d !} \frac{(\Omega / 2 \pi)^{n+1}}{(n+1) !} .
$$

Then apply equivariant $\operatorname{HRR}$ formula (2.7 ) to datum $(\mathcal{X}, \mathcal{L}, \Phi, \alpha)$, it yields

$$
\chi_{d}^{\alpha}(\mathcal{X}, k \mathcal{L})=\int_{\mathcal{X}} \frac{\Theta_{W}^{d}}{d !} \frac{(\Omega / 2 \pi)^{n+1}}{(n+1) !} \cdot k^{n+1+d}+O\left(k^{n+d}\right)
$$

thus (4.2) follows.

The next step is to show the leading coefficient of $\chi_{d}^{\alpha}(\mathcal{X}, k \mathcal{L})$ equals to $(d+1)$-moment of $\mathrm{DH}$ measure $\mathrm{DH}(\mathcal{X}, \mathcal{L})$. For this, we need the associated filtration of section ring introduced by Witt Nyström [41]. 
4.2. Filtrations of the section ring. The references of this subsection are $[6,7,41]$.

Definition 4.5 (Filtrations). Let $(M, L)$ be a polarized manifold. We call $\mathcal{F}=\left\{F^{t} H^{0}(k L)\right\}_{t \in \mathbb{R}, k \geq 0}$ is a filtration of the section ring $R(M, L)=\bigoplus_{k>0} H^{0}(M, k L)$, iffor each $k,\left\{F^{t} H^{0}(k L)\right\}_{t \in \mathbb{R}}$ is a family of subspaces of $H^{0}(M, k L)$ and nonincreasing and left-continuous in $t$. A filtration $\mathcal{F}$ is called admissible if it is

(1) multiplicative: $F^{t} H^{0}(k L) \cdot F^{s} H^{0}(l L) \subset F^{t+s} H^{0}((k+l) L)$;

(2) pointwise left-bounded: for each $k, F^{t} H^{0}(k L)=H^{0}(k L)$ for t small enough;

(3) linear right-bounded: $\exists C>0$ such that $F^{t} H^{0}(k L)=\{0\}$ for $t>C k$ and $k \geq 0$.

Remark 4.6. Since $R(M, L)$ is finitely generated, by [6] Lemma 1.5, any multiplicative and pointwise left-bounded filtration is automatically linear left-bounded, i.e. there exists $C>0$ such that $F^{t} H^{0}(k L)=H^{0}(k L)$ for $t<-C k$ and $k \geq 0$.

Let $\mathcal{F}$ be an admissible filtration of section ring. For each $k$, to study the jumping numbers of filtration, we consider probability measure

$$
\mathfrak{m}_{k}:=-\frac{d}{d \lambda}\left(\frac{1}{N_{k}} \operatorname{dim} F^{k \lambda} \mathrm{H}^{0}(k L)\right),
$$

called the weight distribution. The linear boundness of $\mathcal{F}$ implies that $\left\{\mathfrak{m}_{k}\right\}$ have uniformly bounded support. Its weak limit can be obtained in the following way. For $\lambda \in \mathbb{R}$, we define a graded subalgebra $R^{(\lambda)}$ of $R(M, L)$,

$$
R^{(\lambda)}:=\bigoplus_{k \geq 0} F^{k \lambda} \mathrm{H}^{0}(k L)
$$

Its volume is defined by

$$
\operatorname{vol}\left(R^{(\lambda)}\right):=\limsup _{k \rightarrow \infty} \frac{1}{N_{k}} \operatorname{dim} F^{k \lambda} \mathrm{H}^{0}(k L) .
$$

By the linear boundness, $\operatorname{vol}\left(R^{(\lambda)}\right)=0$ when $\lambda \gg 0$ and $\operatorname{vol}\left(R^{(\lambda)}\right)=1$ when $\lambda \ll 0$.

Theorem 4.7 (see Theorem 5.3 [7]). For an admissible filtration $\mathcal{F}$ of the section ring $R(M, L)$, the sequence of weight distributions $\left\{\mathfrak{m}_{k}\right\}$ weakly converge to $-\frac{d}{d \lambda} \operatorname{vol}\left(R^{(\lambda)}\right)$, where the derivative is taken in the sense of distributions.

We call this weak limit of $\mathfrak{m}_{k}$ the limit measure of admissible filtration $\mathcal{F}$, and denote it by $\operatorname{LM}(\mathcal{F})$.

4.3. Filtrations associated to test-configurations . In [41], Witt Nyström define an admissible filtration of $R(M, L)$ for a test-configuration for $(M, L)$.

Let $(\mathcal{X}, \mathcal{L})$ be an ample test-configuration for $(M, L)$. We use $\left.(\mathcal{X}, \mathcal{L})\right|_{\mathbb{C}}$ to denote the restricted family over $\mathbb{C}$. For each $\mu \in \mathbb{Z}$ and $k \geq 0$, let $F^{\mu} \mathrm{H}^{0}(k L)$ be the image of following restriction map,

$$
\mathrm{H}^{0}\left(\left.\mathcal{X}\right|_{\mathbb{C}}, k \mathcal{L}\right)_{\mu} \hookrightarrow \mathrm{H}^{0}\left(\mathcal{X}_{1}, k \mathcal{L}\right)=\mathrm{H}^{0}(M, k L) .
$$

For $s \in \mathrm{H}^{0}(M, k L)$, let $\bar{s}$ be the equivariant (w.r.t. action $\alpha$ ) extension on $\left.\mathcal{X}\right|_{\mathbb{C}^{*}}$. Then we have an equivalent description:

$$
F^{\mu} \mathrm{H}^{0}(k L)=\left\{s \in \mathrm{H}^{0}(M, k L) \mid \tau^{-\mu} \cdot \bar{s} \in \mathrm{H}^{0}\left(\left.\mathcal{X}\right|_{\mathbb{C}}, k \mathcal{L}\right)\right\} .
$$

Namely, $F^{\mu} \mathrm{H}^{0}(k L)$ is constituted of $s$ such that $\tau^{-\mu} \cdot \bar{s}$ can be extended onto $\left.\mathcal{X}\right|_{\mathbb{C}}$. We can consider the restriction map to $\mathcal{X}_{0}$,

$$
F^{\mu} \mathrm{H}^{0}(k L) \longrightarrow \mathrm{H}^{0}\left(\mathcal{X}_{0}, k \mathcal{L}_{0}\right)_{\mu},\left.s \mapsto\left(\tau^{-\mu} \bar{s}\right)\right|_{\mathcal{X}_{0}} .
$$


Since its kernel is $F^{\mu+1} \mathrm{H}^{0}(k L)$, we have

$$
F^{\mu} \mathrm{H}^{0}(k L) / F^{\mu+1} \mathrm{H}^{0}(k L) \cong \mathrm{H}^{0}\left(\mathcal{X}_{0}, k \mathcal{L}_{0}\right)_{\mu} .
$$

Definition 4.8 (associated filtrations). For an ample test-configuration $(\mathcal{X}, \mathcal{L})$ of $(M, L)$, define the associated filtration is

$$
\mathcal{F}(\mathcal{X}, \mathcal{L})=\left\{F^{\lceil t\rceil} H^{0}(k L)\right\}_{t \in \mathbb{R}, k \geq 0}
$$

where $F^{\lceil t\rceil} H^{0}(k L)$ is given by (4.4). By [41], it is an admissible filtration of $R(M, L)$.

By (4.5) and (2.1), we see the limit measure of $\mathcal{F}(\mathcal{X}, \mathcal{L})$ is $\mathrm{DH}$ measure $\mathrm{DH}(\mathcal{X}, \mathcal{L})$, and Theorem 4.7 implies

$$
\operatorname{DH}(\mathcal{X}, \mathcal{L})=-\frac{d}{d \lambda} \operatorname{vol}\left(R^{(\lambda)}\right)
$$

Remark 4.9. Consider the relation between $\mathcal{F}(\mathcal{X}, \mathcal{L})$ and sections $H^{0}(\mathcal{X}, k \mathcal{L})$ over the compactified total space. For $s \in H^{0}(k L)$, since action $\alpha$ on $(\mathcal{X}, \mathcal{L})_{\infty}$ is trivial, it is easy to see $\tau^{-\mu} \cdot \bar{s}$ can be extended onto $\mathcal{X}_{\infty}$ only if $\mu \geq 0$. Hence the relation is

$$
H^{0}(\mathcal{X}, k \mathcal{L})_{\mu}=F^{\mu} H^{0}(k L) \text {, if } \mu \geq 0 \text { and } H^{0}(\mathcal{X}, k \mathcal{L})_{\mu}=0 \text {, if } \mu<0 \text {. }
$$

Thus if only take the sections over the compactified space, we can not obtain the negative part of $\mathcal{F}(\mathcal{X}, \mathcal{L})$.

\subsection{Proof of Theorem 1.5.}

Proof. It is sufficient to show that for any integer $d \geq 0$, we have

$$
\lim _{t \rightarrow \infty} \frac{1}{V} \int_{M} \frac{\left(\dot{u}_{t}\right)^{d+1}}{(d+1) !} \omega_{u_{t}}^{n}=\int_{\mathbb{R}} \frac{\lambda^{d+1}}{(d+1) !} d \operatorname{DH}(\mathcal{X}, \mathcal{L}) .
$$

Using Proposition 4.4 as a bridge, we only need to connect the leading coefficient of $\chi_{d}^{\alpha}(\mathcal{X}, k \mathcal{L})$ to $\operatorname{DH}(\mathcal{X}, \mathcal{L})$. The associated filtration $\mathcal{F}(\mathcal{X}, \mathcal{L})$ can connect them. But there are two issues, one is that $\mathcal{F}(\mathcal{X}, \mathcal{L})$ does not involve $\mathrm{H}^{q}(\mathcal{X}, k \mathcal{L})_{\mu}(q>0)$ but it appears in $\chi_{d}^{\alpha}$; another issue has been mentioned in Remark 4.9, the negative part of $\mathcal{F}(\mathcal{X}, \mathcal{L})$ is lost if we only take sections over $\mathcal{X}$. Fortunately, by the relative ampleness of $\mathcal{L}$, both issues can be avoided.

Take a large integer $c$, let $\mathcal{L}_{c}:=\mathcal{L}+\pi^{*} \mathcal{O}_{\mathbb{P}^{1}}(c)$ equipped the product $\mathbb{C}^{*}$-action (action on $\mathcal{O}_{\mathbb{P}^{1}}(c)$ is defined in Remark 2.2). Actually, $\left(\mathcal{X}, \mathcal{L}_{c}\right)$ is the compactification of $\left.(\mathcal{X}, \mathcal{L})\right|_{\mathbb{C}}$ with modified structure action by multiplying character $\tau^{c}$.

We endow $\mathcal{L}_{c}$ with metric $\Phi_{c}:=\Phi+c \cdot \pi^{*} \phi_{F S}$, here $\phi_{F S}$ is the Fubini-Study metric on $\mathcal{O}_{\mathbb{P}^{1}}(-1)$. The curvature current is $\Omega_{c}:=\Omega+c \pi^{*} \omega_{F S}, \omega_{F S}=i \partial \bar{\partial} \log \left(1+|\tau|^{2}\right)$. We can check the Hamiltonian function associated to datum $\left(\mathcal{X}, \mathcal{L}_{c}, \Phi_{c}\right)$ is

$$
\Theta_{W, c}:=\Theta_{W}+\frac{c}{1+|\tau|^{2}} .
$$

It vanishes on $\mathcal{X}_{\infty}$ as we expected.

Now applying Proposition 4.4 to datum $\left(\mathcal{X}, \mathcal{L}_{c}, \Phi_{c}\right)$, it yields

$$
\lim _{t \rightarrow \infty} \frac{1}{V} \int_{M} \frac{1}{(d+1) !}\left(\dot{u}_{t}+\frac{c}{1+e^{-t}}\right)^{d+1} \omega_{u_{t}}^{n}=\lim _{k \rightarrow \infty} \frac{\chi_{d}^{\alpha}\left(\mathcal{X}, k \mathcal{L}_{c}\right)}{k^{d+1} N_{k}} .
$$

Since $\mathcal{L}$ is relatively ample, we can choose $c$ is sufficiently large such that $\mathcal{L}_{c}$ is ample over $\mathcal{X}$. Then for $k \gg 1$, we have

$$
\chi_{d}^{\alpha}(\mathcal{X}, k \mathcal{L})=\sum_{\mu \in \mathbb{Z}} \frac{\mu^{d}}{d !} \cdot \operatorname{dim} \mathrm{H}^{0}\left(\mathcal{X}, k \mathcal{L}_{c}\right)_{\mu} .
$$


Let $F^{\mu} \mathrm{H}^{0}(k L)$ be the filtration associated to $(\mathcal{X}, \mathcal{L})$, then it is easy to see the filtration associated to $\left(\mathcal{X}, \mathcal{L}_{c}\right)$ is

$$
\left\{F^{\mu-k c} \mathrm{H}^{0}(k L)\right\}_{\mu \in \mathbb{Z}} .
$$

By the relation (4.7) for $\left(\mathcal{X}, \mathcal{L}_{c}\right)$, we have

$$
\begin{aligned}
\frac{1}{k^{d+1} N_{k}} \chi_{d}^{\alpha}\left(\mathcal{X}, k \mathcal{L}_{c}\right) & =\frac{1}{k^{d+1} N_{k}} \sum_{\mu \geq 0} \frac{\mu^{d}}{d !} \cdot \operatorname{dim} F^{\mu-k c} \mathrm{H}^{0}(k L) \\
(\text { let } \mu=k \lambda) & =\sum_{0 \leq \lambda \in \frac{1}{k} \mathbb{Z}} \frac{\lambda^{d}}{d !} \cdot \frac{1}{N_{k}} \operatorname{dim} F^{k(\lambda-c)} \mathrm{H}^{0}(k L) \cdot \frac{1}{k} .
\end{aligned}
$$

Let $k \rightarrow \infty$, by definition (4.3), above sum converges to

$$
\int_{0}^{\infty} \frac{\lambda^{d}}{d !} \operatorname{vol}\left(R^{(\lambda-c)}\right) d \lambda=\int_{-c}^{\infty} \frac{(\lambda+c)^{d}}{d !} \operatorname{vol}\left(R^{(\lambda)}\right) d \lambda .
$$

We take $c$ to be large enough such that $\operatorname{suppDH}(\mathcal{X}, \mathcal{L}) \subset[-c+1, \infty)$. Integrating by parts and use (4.6), it further equals to

$$
\int_{-c}^{\infty} \frac{(\lambda+c)^{d+1}}{(d+1) !} d \mathrm{DH}(\mathcal{X}, \mathcal{L})=\int_{\mathbb{R}} \frac{(\lambda+c)^{d+1}}{(d+1) !} d \mathrm{DH}(\mathcal{X}, \mathcal{L}) .
$$

In a summary, we have showed that

$$
\lim _{t \rightarrow \infty} \frac{1}{V} \int_{M} \frac{1}{(d+1) !}\left(\dot{u}_{t}+\frac{c}{1+e^{-t}}\right)^{d+1} \omega_{u_{t}}^{n}=\int_{\mathbb{R}} \frac{(\lambda+c)^{d+1}}{(d+1) !} d \mathrm{DH}(\mathcal{X}, \mathcal{L})
$$

for all integer $c \gg 1$. Expand the LHS, we see it is a polynomial in $c$, so it is for the RHS. Hence the constant terms of two sides are same, that is (4.8).

\section{Mabuchi SOlitons AND RElative D-SEMistability}

5.1. Extremal vector fields. Let $M$ be a Fano manifold, set $L=-K_{M}$. There is a canonical lifting of action $\operatorname{Aut}(M) \hookrightarrow \operatorname{Aut}(M, L)$. By Kodaira's imbedding, the induced homomorphism $\operatorname{Aut}(M, L) \rightarrow \mathrm{GL}\left(\mathrm{H}^{0}(M, k L)\right)$ will be injective when $k \gg 1$, thus $\operatorname{Aut}(M)$ is a linear algebraic group.

Let $K \subset \operatorname{Aut}^{0}(M)$ be a maximal compact subgroup with Lie algebra k. By CartanIwasawa-Malcev theorem, $K$ is connected and unique up to a conjugation. Let $G \subset$ $\operatorname{Aut}^{0}(M)$ be its complexification with Lie algebra $\mathfrak{k} \otimes \mathbb{C}$. In this paper, elements in Lie algebra are $(1,0)$-type holomorphic vector fields, and the exponential map is

$$
\mathfrak{k} \rightarrow K, X \mapsto \exp (\operatorname{Im} X),
$$

the latter is the time- 1 map generated by $\operatorname{Im} X$.

Then we take a $K$-invariant reference metric $\omega \in 2 \pi c_{1}(M)$, let

$$
\mathcal{H}_{\omega}^{K}=\left\{u \in C^{\infty}(M) \mid \omega_{u}>0, \operatorname{Im} X . u=0, \forall X \in \mathfrak{k}\right\}
$$

be the space of $K$-invariant Kähler potentials. Since $K$ is maximal, for each $u \in \mathcal{H}_{\omega}^{K}$, the identity component of the isometry group of $\omega_{u}$ is exactly $K$.

For any $X \in \mathfrak{k}$, let $\theta_{X}(\omega)$ be the normalized Hamiltonian function of $X$ w.r.t. $\omega$, which satisfies $\iota_{X} \omega=i \bar{\partial} \theta_{X}(\omega)$ and $\int \theta_{X}(\omega) \omega^{n}=0$. For any $u \in \mathcal{H}_{\omega}^{K}$, let $\theta_{X}(u):=\theta_{X}(\omega)+$ $X(u)$, we have $\iota_{X} \omega_{u}=i \bar{\partial} \theta_{X}(u)$. By the below proposition, $\theta_{X}(u)$ is the normalized Hamiltonian function of $X$ w.r.t. $\omega_{u}$. 
Proposition 5.1. (1) For each $X \in \mathfrak{k}$, the pushforward of measure $\omega_{u}^{n}$ under the map $\theta_{X}(u): M \rightarrow \mathbb{R}$ is independent of $u \in \mathcal{H}_{\omega}^{K}$. In particular, its barycenter is $\int \theta_{X}(u) \omega_{u}^{n}=$ $\int \theta_{X}(\omega) \omega^{n}=0$.

(2) For each $X, Y \in \mathfrak{k}$, the pushforward of measure $\omega_{u}^{n}$ under the map $\left(\theta_{X}(u), \theta_{Y}(u)\right)$ : $M \rightarrow \mathbb{R}^{2}$ is also independent of $u \in \mathcal{H}_{\omega}^{K}$.

Proof. For (1), we only need to show that for any $f \in C^{1}(\mathbb{R})$ and $u \in \mathcal{H}_{\omega}^{K}$, we have

$$
\int_{M} f\left(\theta_{X}(u)\right) \omega_{u}^{n}=\int_{M} f\left(\theta_{X}(\omega)\right) \omega^{n} .
$$

For $t \in[0,1], t u \in \mathcal{H}_{\omega}^{K}$, we compute

$$
\begin{aligned}
\frac{d}{d t} \int_{M} f\left(\theta_{X}(t u)\right) \omega_{t u}^{n} & =\int f^{\prime} X(u) \omega_{t u}^{n}+\int f\left(\theta_{X}(t u)\right) i \partial \bar{\partial} u \wedge n \omega_{t u}^{n-1} \\
& =\int f^{\prime} X(u) \omega_{t u}^{n}+\int f^{\prime} i \bar{\partial} \theta_{X}(t u) \wedge \partial u \wedge n \omega_{t u}^{n-1} \\
& =\int f^{\prime} X(u) \omega_{t u}^{n}+\int f^{\prime} \iota_{X} \omega_{t u} \wedge \partial u \wedge n \omega_{t u}^{n-1}=0
\end{aligned}
$$

The proof of (2) is similar with (1).

Definition 5.2 (DH measure). Let $K \subset A u t^{0}(M)$ be a maximal compact subgroup with Lie algebra $\mathfrak{k}$. Since the part (1) of above proposition, we denote the pushforward measure by

$$
D H_{K}(X):=\theta_{X}(\omega)_{\#} \frac{\omega^{n}}{V}
$$

which is independent of the choice of $K$-invariant metric $\omega \in 2 \pi c_{1}(M)$. We call it the $D H$ measure of vector field $X \in \mathfrak{k}$.

For $X, Y \in \mathfrak{k}$ and any $u \in \mathcal{H}_{\omega}^{K}$, we define

$$
B_{K}(X, Y):=\frac{1}{V} \int_{M} \theta_{X}(u) \theta_{Y}(u) \omega_{u}^{n} .
$$

By part (2) of the above proposition, it is independent of the choice of $u$ and an inner product on $\mathfrak{k}$. This is the restriction of the bilinear form introduced by Futaki-Mabuchi [15].

Remark 5.3. Let $K^{\prime}=g K g^{-1}, g \in A u t^{0}(M)$ be another choice of maximal compact subgroups. Then $\mathfrak{k}^{\prime}=g_{*} \mathfrak{k}$ and $\omega^{\prime}=\left(g^{-1}\right)^{*} \omega$ is $K^{\prime}$-invariant. For any $X \in \mathfrak{k}$, let $X^{\prime}=$ $g_{*} X \in \mathfrak{k}^{\prime}$, it is easy to see that

$$
\theta_{X^{\prime}}\left(\omega^{\prime}\right)=\theta_{X}(\omega) \circ g^{-1}
$$

This follows that $D H_{K^{\prime}}\left(X^{\prime}\right)=D H_{K}(X)$. For the inner product, let $X, Y \in \mathfrak{k}$ and $X^{\prime}=$ $g_{*} X, Y^{\prime}=g_{*} Y \in \mathfrak{k}^{\prime}$, we have $B_{K^{\prime}}\left(X^{\prime}, Y^{\prime}\right)=B_{K}(X, Y)$.

For a holomorphic vector field $X$, let $\omega \in 2 \pi c_{1}(M)$ with Ricci potential $h_{\omega}$, the Futaki invariant is defined by

$$
F(X):=\frac{1}{V} \int X\left(h_{\omega}\right) \omega^{n} .
$$

It is independent of the choice of $\omega$. For a maximal compact subgroup $K \subset \operatorname{Aut}^{0}(M)$, by taking $\omega$ to be $K$-invariant, we see the restriction of $F$ on $\mathfrak{k}$ is real-valued. 
Definition 5.4 (extremal vector fields and invariant $\vartheta(M)$ ). Let $K \subset A u t^{0}(M)$ be a maximal compact subgroup, there is a unique element $Z_{K} \in \mathfrak{k}$ such that

$$
F(X)=-B_{K}\left(X, Z_{K}\right), \text { for } \forall X \in \mathfrak{k} \text {. }
$$

We call $Z_{K}$ the extremal vector field associated to $K$. If $K^{\prime}=g K g^{-1}, g \in A u t^{0}(M)$ is another maximal compact subgroup, since $F\left(g_{*} X\right)=F(X)$, we have $Z_{K^{\prime}}=g_{*} Z_{K}$. Then by Remark 5.3, $D H_{K}\left(Z_{K}\right)$ is independent of the choice of $K$. Thus we omit " $K$ " and denote it by

$$
D H(M):=\theta_{Z_{K}}(\omega)_{\#} \frac{\omega^{n}}{V},
$$

where $\omega$ is any $K$-invariant metric $\omega \in 2 \pi c_{1}(M) . D H(M)$ is an invariant for Fano manifolds. In this paper, we mostly concern the invariant

$$
\vartheta(M):=\max _{M} \theta_{Z_{K}}(\omega)=\sup \operatorname{suppDH}(M)-\int_{\mathbb{R}} \lambda d D H(M),
$$

where the barycenter of $\mathrm{DH}(M)$ is the origin. It had been introduced by Mabuchi [30] with notation $\alpha_{M}$.

The extremal vector field has many important properties. By [15], $Z_{K}$ belongs to the center of $\mathfrak{k}$ and its imaginary part generates a circle action, which we call the extremal action.

There is another equivalent definition of $Z_{K}$ in [15] in terms of scalar curvatures. Fix $K \subset \operatorname{Aut}^{0}(M)$, and take a $K$-invariant metric $\omega$. We endow the space $C^{\infty}(M, \mathbb{R})$ with $L^{2}$-inner product $\frac{1}{V} \int f_{1} f_{2} \omega^{n}$, and then consider the orthogonal projection to the subspace

$$
P_{\omega}: C^{\infty}(M, \mathbb{R}) \rightarrow\left\{\theta_{X}(\omega) \mid X \in \mathfrak{k}\right\} .
$$

Since for any $X \in \mathfrak{k}$, we have

$$
F(X)=-\int h_{\omega} \mathcal{L}_{X} \frac{\omega^{n}}{V}=-\int h \triangle \theta_{X}(\omega) \frac{\omega^{n}}{V}=-\int \theta_{X}(\omega)(S(\omega)-n) \frac{\omega^{n}}{V} .
$$

It follows that $\theta_{Z_{K}}(\omega)=P_{\omega}(S(\omega)-n)$, thus

$$
Z_{K}=\operatorname{grad}_{\omega} P_{\omega}(S(\omega)-n)
$$

Moreover, Theorem 2.1 in [30] says $P_{\omega}(S(\omega)-n)=P_{\omega}\left(1-e^{h_{\omega}}\right)$, hence $Z_{K}$ also can be defined by

$$
Z_{K}=\operatorname{grad}_{\omega} P_{\omega}\left(1-e^{h_{\omega}}\right) .
$$

5.2. Mabuchi solitons. There is an analogue of Calabi energy. Let $\omega \in 2 \pi c_{1}(M)$ be a reference metric and $\mathcal{H}_{\omega}=\left\{u \in C^{\infty}(M) \mid \omega_{u}>0\right\}$. The Ding energy is defined by

$$
\operatorname{Din}(u)=\frac{1}{V} \int_{M}\left(e^{h(u)}-1\right)^{2} \omega_{u}^{n}, u \in \mathcal{H}_{\omega},
$$

where $h(u)$ is the Ricci potential of $\omega_{u}$.

Definition 5.5. If $u \in \mathcal{H}_{\omega}$ is a critical point of Ding energy, then we say the associated metric $\omega_{u}$ is a Mabuchi soliton.

In this sense, Mabuchi solitons are analogues of extremal metrics which are critical points of Calabi energy. This definition is equivalent to that one given by Mabuchi [30].

Theorem 5.6. [42] $\omega_{u}$ is a Mabuchi soliton if and only if the vector field $\operatorname{grad}_{\omega_{u}}\left(1-e^{h(u)}\right)$ is holomorphic. 
Moreover, Mabuchi soliton is unique modulo the action of $\operatorname{Aut}^{0}(M)$, see Theorem $\mathrm{C}$ in [32] and Corollary B in [31]. As with extremal metrics, the intersection of the isometry group of a Mabuchi soliton with $\operatorname{Aut}^{0}(M)$ is maximally compact in $\operatorname{Aut}^{0}(M)$, see Theorem $\mathrm{D}$ in [31]. Clearly, if $M$ admits a Mabuchi soliton, then for any maximal compact subgroup $K$, there exists a $K$-invariant Mabuchi soliton, since all maximal compact subgroups are conjugated with each other. Hence in the search for a Mabuchi soliton, we firstly fix a maximal compact subgroup $K \subset \operatorname{Aut}^{0}(M)$, and take the reference metric $\omega \in 2 \pi c_{1}(M)$ is $K$-invariant. Let $\mathcal{H}_{\omega}^{K}$ be the space of $K$-invariant Kähler potentials.

Suppose $u \in \mathcal{H}_{\omega}^{K}$ such that $X=\operatorname{grad}_{u}\left(1-e^{h(u)}\right)$ is holomorphic. Since $\theta_{X}(u)=$ $1-e^{h(u)}$ is real-valued, $\operatorname{Im} X$ preserves $\omega_{u}$. Since the isometry group of $\omega_{u}$ is $K$, so $X \in \mathfrak{k}$. Then by (5.1) we conclude $X=Z_{K}$. After adding $u$ by a constant, we see $u$ satisfies a Monge-Ampère type equation

$$
\left(1-\theta_{Z}(u)\right) \omega_{u}^{n}=e^{h_{\omega}-u} \omega^{n} .
$$

A necessary condition for it admits a smooth solution is $\max _{M} \theta_{Z}(u)<1$, i.e. $\vartheta(M)<1$.

5.3. Modified Ding functionals . Fix $K \subset \operatorname{Aut}^{0}(M)$ and a $K$-invariant reference metric $\omega \in 2 \pi c_{1}(M)$. Let $Z=Z_{K}$ be the associated extremal vector field. We define

$$
\mathcal{H}_{\omega}^{Z}=\left\{u \in C^{\infty}(M) \mid \omega_{u}>0, \operatorname{Im} Z . u=0\right\} \supset \mathcal{H}_{\omega}^{K} .
$$

Then $\theta_{Z}(u)$ is real-valued for each $u \in \mathcal{H}_{\omega}^{Z}$.

Definition 5.7. (1) The modified Monge-Ampère Energy $E_{Z}: \mathcal{H}_{\omega}^{Z} \rightarrow \mathbb{R}$ is defined such that $E_{Z}(0)=0$ and

$$
\left.\delta E_{Z}\right|_{u}(\delta u)=\frac{1}{V} \int_{M} \delta u\left(1-\theta_{Z}(u)\right) \omega_{u}^{n} .
$$

(2) The modified Ding functional $D_{Z}: \mathcal{H}_{\omega}^{Z} \rightarrow \mathbb{R}$ is defined by

$$
D_{Z}(u):=-E_{Z}(u)+L(u), L(u):=-\log \left(\frac{1}{V} \int_{M} e^{h_{\omega}-u} \omega^{n}\right) .
$$

When $Z=0, D_{Z}$ is the ordinary Ding functional.

For $u \in \mathcal{H}_{\omega}^{Z}$, integrating along the path $u_{t}=t u$ for $t \in[0,1]$, we have

$$
E_{Z}(u)=\int_{0}^{1} d t \int_{M} u\left(1-\theta_{Z}(\omega)-t \cdot Z u\right)\left(t \omega_{u}+(1-t) \omega\right)^{n} .
$$

Expanding the volume form and integrating $t$, we can obtain an explicit formula for $E_{Z}$.

By definition, the variation of $D_{Z}$ is

$$
\left.\delta D_{Z}\right|_{u}(\delta u)=-\frac{1}{V} \int_{M} \delta u\left(1-e^{h(u)}-\theta_{Z}(u)\right) \omega_{u}^{n},
$$

thus its critical points are Mabuchi solitons.

Moreover, $D_{Z}$ can be extended to the space of $\operatorname{Im} Z$-invariant bounded psh metrics on $K_{M}^{-1}$, which is denoted by

$$
\mathcal{H}_{b}^{Z}=\left\{\phi \mid \operatorname{Im} Z \text {-invariant, bounded psh metric on } K_{M}^{-1}\right\}
$$

By [5] Proposition 2.17, $E_{Z}$ is convex along bounded sub-geodesics in $\mathcal{H}_{b}^{Z}$ and affine along geodesics. Since $L(u)$ is also convex along bounded geodesics due to Berndtsson, thus $D_{Z}$ is convex along bounded geodesics. 
5.4. Modified K-energies. We only consider extremal metrics in $c_{1}(M)$ for a Fano manifold $M$. Let $K \subset \operatorname{Aut}^{0}(M)$ be a maximal compact subgroup with associated extremal vector field $Z$. The modified K-energy $M_{Z}: \mathcal{H}_{\omega}^{Z} \rightarrow \mathbb{R}$ is defined such that $M_{Z}(0)=0$ and

$$
\left.\delta M_{Z}\right|_{u}(\delta u)=-\frac{1}{V} \int_{M} \delta u\left(S\left(\omega_{u}\right)-n-\theta_{Z}(u)\right) \omega_{u}^{n} .
$$

Integrating along a smooth path $\left\{u_{t}\right\}$ from 0 to $u$ (e.g. $u_{t}=t u$ ), we have

$$
M_{Z}(u)=-\frac{1}{V} \int_{0}^{1} d t \int_{M} \dot{u}_{t}\left(S\left(\omega_{u_{t}}\right)-n-1\right) \omega_{u_{t}}^{n}-E_{Z}(u)
$$

We derive the Chen-Tian formula of $M_{Z}$. Let $\Omega_{u}=e^{h_{\omega}-u} \omega^{n}$, then $\operatorname{Ric}\left(\Omega_{u}\right)=\omega_{u}$. Note that $-\int_{M} \dot{u}_{t}\left(S\left(\omega_{u_{t}}\right)-n\right) \omega_{u_{t}}^{n}$ is equal to

$$
\begin{aligned}
& -n \int \dot{u}_{t}\left(\operatorname{Ric}\left(\omega_{u_{t}}\right)-\operatorname{Ric}\left(\Omega_{u_{t}}\right)\right) \wedge \omega_{u_{t}}^{n-1}=n \int \dot{u_{t}} i \partial \bar{\partial} \log \frac{\omega_{u_{t}}^{n}}{\Omega_{u_{t}}} \wedge \omega_{u_{t}}^{n-1} \\
= & \int \log \frac{\omega_{u_{t}}^{n}}{\Omega_{u_{t}}} \frac{d}{d t} \omega_{u_{t}}^{n}=\frac{d}{d t}\left[\int \log \left(\frac{\omega_{u_{t}}^{n}}{\Omega_{u_{t}}}\right) \omega_{u_{t}}^{n}\right]-\int \dot{u}_{t} \omega_{u_{t}}^{n} .
\end{aligned}
$$

Putting this back and integrate over $t$, we obtain

$$
\begin{aligned}
M_{Z}(u) & =\frac{1}{V} \int \log \left(\frac{\omega_{u}^{n}}{\Omega_{u}}\right) \omega_{u}^{n}+\frac{1}{V} \int h_{\omega} \omega^{n}-E_{Z}(u) \\
& =D_{Z}(u)+\int \log \left(\frac{\omega_{u}^{n} / V}{\Omega_{u} / \int \Omega_{u}}\right) \frac{\omega_{u}^{n}}{V}+\frac{1}{V} \int h_{\omega} \omega^{n} \\
& \geq D_{Z}(u)+\frac{1}{V} \int h_{\omega} \omega^{n}
\end{aligned}
$$

where the entropy term is nonnegative by Jensen's inequality.

Remark 5.8. The above inequality for $M_{Z}$ and $D_{Z}$ implies an implication relation between extremal metrics and Mabuchi solitons. If Fano manifold $M$ admits a Mabuchi soliton, by [29] or [16] $D_{Z}$ is proper in the sense therein, the above inequality implies $M_{Z}$ is also proper. Then by the recent breakthrough by Chen-Cheng [9] (also see [19]), $M$ admits extremal metrics. Conversely, toric 3-fold $\mathbb{P}\left(\mathcal{O}_{\mathbb{P}^{2}} \oplus \mathcal{O}_{\mathbb{P}^{2}}(2)\right)$ does not admit Mabuchi solitons (it is relative D-unstable, see [34]), but as a projective bundle it admits extremal metrics by the construction in [2].

5.5. Limit slopes and relative D-semistability . By [15] Theorem $\mathrm{F}, \operatorname{Im} Z$ generates a circle action, then there exists a minimum $\tau=\tau_{Z}>0$ such that $\exp 4 \pi \tau \cdot \operatorname{Im} Z=I d$. Thus $\tau Z$ generates a $\mathbb{C}^{*}$-action $\beta_{\tau Z}$ satisfying

$$
\beta_{\tau Z}\left(e^{-\frac{1}{2}(t+i s)}\right)=\exp (t \cdot \tau \operatorname{Re} Z-s \cdot \tau \operatorname{Im} Z) .
$$

We call it the extremal action associated to maximal compact subgroup $K$. We use " $\beta_{Z}$ " to denote the formal $\mathbb{C}^{*}$-action generated by $Z$.

Assume $(\mathcal{X}, \mathcal{L})$ is a $\mathbb{C}^{*}$-equivariant test-configuration with fiberwise $\mathbb{C}^{*}$-action $\beta_{\tau Z}$, we define the inner product of the structure $\mathbb{C}^{*}$-action $\alpha$ and the formal $\mathbb{C}^{*}$-action $\beta_{Z}$ is

$$
\left\langle\alpha, \beta_{Z}\right\rangle:=\frac{1}{\tau}\left\langle\alpha, \beta_{\tau Z}\right\rangle .
$$

For simplicity, we will not explicitly mention the period $\tau$ and just take $\beta_{Z}$ to be a real $\mathbb{C}^{*}$-action. 
Proposition 5.9. Let $(\mathcal{X}, \mathcal{L})$ be a $\mathbb{C}^{*}$-equivariant ample test-configuration for $\left(M,-K_{M}\right)$ with structure action $\alpha$ and fiberwise extremal action $\beta_{Z}$. Let $\Phi$ be a $\alpha\left(\mathbb{S}^{1}\right) \times \beta\left(\mathbb{S}^{1}\right)$ invariant metric on $\mathcal{L}$ satisfying condition $(B)$ in Definition 3.3. Pulling back by $\alpha, \Phi$ induces a ray $\left\{u_{t}\right\}_{t \geq 0} \subset \mathcal{H}_{b}^{Z}$. Then we have

$$
\lim _{t \rightarrow \infty} \frac{d}{d t} E_{Z}\left(u_{t}\right)=E^{N A}(\mathcal{X}, \mathcal{L})-\left\langle\alpha, \beta_{Z}\right\rangle
$$

and

$$
\lim _{t \rightarrow \infty} \frac{d}{d t} D_{Z}\left(u_{t}\right)=D^{N A}(\mathcal{X}, \mathcal{L})+\left\langle\alpha, \beta_{Z}\right\rangle
$$

Proof. The first identity follows from Theorem 3.7 and Theorem 1.5. Then by the formula of limit slope of $L\left(u_{t}\right)$ due to Berman [3], the second identity follows.

Definition 5.10 (relative D-semistability). Let $M$ be a Fano manifold. Let $K \subset A u t^{0}(M)$ be a compact subgroup with complexification $G=K^{\mathbb{C}} \subset A u t^{0}(M)$. Assume $K^{m} \supset K$ is a maximal compact subgroup such that $Z:=Z_{K^{m}}$ belongs to Lie $(K)$. In this paper, we take $G$ to be a torus.

(1) Let $(\mathcal{X}, \mathcal{L})$ be a $G$-equivariant ample test-configuration for $\left(M,-K_{M}\right)$, the relative Berman-Ding invariant is defined by

$$
D_{Z}^{N A}(\mathcal{X}, \mathcal{L}):=D^{N A}(\mathcal{X}, \mathcal{L})+\left\langle\alpha, \beta_{Z}\right\rangle
$$

where $\left\langle\alpha, \beta_{Z}\right\rangle$ is defined by taking $(\mathcal{X}, \mathcal{L})$ as a $\mathbb{C}^{*}$-equivariant test-configuration via $\beta_{Z}$ : $\mathbb{C}^{*} \rightarrow G$.

(2) We say $M$ is D-semistable relative to $G$, iffor any $G$-equivariant ample test-configuration $(\mathcal{X}, \mathcal{L})$, we always have $D_{Z}^{N A}(\mathcal{X}, \mathcal{L}) \geq 0$.

The existence of Mabuchi solitons can imply that $M$ is D-semistable relative to $G$. The argument is similar to the KE situation [3]. If $M$ admits a $K$-invariant Mabuchi solitons, we consider the Phong-Sturm's geodesic ray $\left\{u_{t}\right\}$ associated to a $G$-equivariant testconfigurations and starting from that Mabuchi solitons. Since $\frac{d}{d t} D_{Z}\left(u_{t}\right)$ is nondecreasing, then we have

$$
0 \leq\left.\frac{d}{d t} D_{Z}\left(u_{t}\right)\right|_{t=0} \leq \lim _{t \rightarrow \infty} \frac{d}{d t} D_{Z}\left(u_{t}\right)=D_{Z}^{N A}(\mathcal{X}, \mathcal{L})
$$

\section{Relative D-Semistability implies $\vartheta(M) \leq 1$} 1.

In this section, we show that $M$ is relatively D-semistable can imply $\vartheta(M)=\sup _{M} \theta_{Z}(\omega) \leq$

Let $T \subset \operatorname{Aut}^{0}(M)$ be a $m$-dimensional complex torus which complexifies compact torus $S$. Suppose $K$ is a maximal compact subgroup containing $S$ and such that the associated extremal vector field $Z$ belongs to $\operatorname{Lie}(S)$. Take a $K$-invariant Kähler metric $\omega \in 2 \pi c_{1}(M)$. Let $\theta$ the normalized Hamiltonian function of $Z$ satisfying $\iota_{Z} \omega=i \bar{\partial} \theta$ and $\int \theta \omega^{n}=0$.

Theorem 6.1. Let $M$ be a Fano manifold, $T \subset A u t^{0}(M)$ be a complex torus. If $M$ is $D$-semistable relative to $T$, then $\vartheta(M) \leq 1$.

The proof is by constructing a specific $T$-equivariant test-configuration: the deformation to normal cone. This kind of test-configurations have been systematically studied in [37]. First we need to choose the center of blowing-up.

Proposition 6.2. There exists a T-fixed point $z_{*} \in M$ attains the maximum value of $\theta$ i.e. $\vartheta(M)$. 
Proof. Since $T$-action can be canonically lifted to $-K_{M}$ and $\omega$ is $S$-invariant, it induces a moment map $\mu: M \rightarrow \triangle$ for $S$-action, where $\triangle \subset \mathbb{R}^{m}$ is a convex set. Since $Z \in \operatorname{Lie}(S)$, $\theta$ can be represented as $l \circ \mu$ by an affine function $l$ on $\mathbb{R}^{m}$. By [1], $\triangle$ is the convex hull of image of $T$-fixed points under $\mu$, thus the maximum of $\left.l\right|_{\triangle}$ can be attained at the image of some $T$-fixed point, say $z_{*}$, it has desired properties.

Proof of Theorem 6.1. Consider the deformation to normal cone of $z_{*}$ (provided by above lemma). First, take product $\left(M \times \mathbb{P}^{1}, p_{1}^{*} K_{M}^{-1}\right)$, the structure action $\alpha$ is the multiplication on $\mathbb{P}^{1}$, the fiberwise $T$-action $\beta$ is the given torus $T \subset \operatorname{Aut}^{0}(M)$. Let

$$
\Pi: \mathcal{X} \rightarrow M \times \mathbb{P}^{1}
$$

be the blowup at point $\left(z_{*}, 0\right)$ with exceptional divisor $P \cong \mathbb{P}^{n}$. Let

$$
\pi=p_{2} \circ \Pi: \mathcal{X} \rightarrow \mathbb{P}^{1}, p=p_{1} \circ \Pi: \mathcal{X} \rightarrow M .
$$

For $c \in \mathbb{Q}_{+}$, define a $\mathbb{Q}$-line bundle $\mathcal{L}_{c}=p^{*} K_{M}^{-1}-c P$ on $\mathcal{X}$. When $c \ll 1, \mathcal{L}_{c}$ is relatively ample. Since action $\alpha$ and $\beta$ fixes $\left(z_{*}, 0\right) \in M \times \mathbb{P}^{1}$, they can be lifted to $\mathcal{X}$. Since $P$ is invariant, they can be further lifted to $\mathcal{L}_{c}$ such that $\alpha$ trivially acts on the fiber over $\infty$ and $\beta$ is the given torus $T \subset \operatorname{Aut}^{0}(M)$ on fibers $\left.\left(\mathcal{X}, \mathcal{L}_{c}\right)\right|_{\tau \neq 0} \cong\left(M, K_{M}^{-1}\right)$.

In a summary, $\left(\mathcal{X}, \mathcal{L}_{c}\right)$ is a family of $T$-equivariant test-configurations for $\left(M,-K_{M}\right)$ with parameter $c \ll 1$. It can be seen as a perturbation of the trivial product test-configuration.

We need to compute $D_{Z}^{N A}\left(\mathcal{X}, \mathcal{L}_{c}\right)$. The $D^{N A}$-part is easy. Since $K_{\mathcal{X} / \mathbb{P}^{1}}=p^{*} K_{M}+n P$, let $B=(n-c) P$, we have $\mathcal{L}_{c}+K_{\mathcal{X} / \mathbb{P}^{1}}=\mathcal{O}(B)$. Note $\mathcal{X}_{0}=\hat{M}+P$, and $\hat{M}$ is the blowup of $M$ at $z_{*}$. The lct-part of $D^{N A}$ is

$$
\operatorname{lct}\left(\mathcal{X},-B ; \mathcal{X}_{0}\right)=\sup \left\{t \in \mathbb{R} \mid\left(\mathcal{X},-B+t \mathcal{X}_{0}\right) \text { is log canonical }\right\} .
$$

Since $\mathcal{X}$ is smooth and $-B+t \mathcal{X}_{0}=t \hat{M}+(c-n+t) P$ is simple normal crossing, thus we have

$$
\operatorname{lct}\left(\mathcal{X},-B ; \mathcal{X}_{0}\right)=\min \{1, n-c+1\}=1, \text { when } c \ll 1 .
$$

On the other hand, $\mathcal{L}_{c}^{n+1}=\left(p^{*} K_{M}^{-1}-c P\right)^{n+1}=-c^{n+1}$. Put these into the definition of $D^{N A}(2.5)$, we have

$$
D^{N A}\left(\mathcal{X}, \mathcal{L}_{c}\right)=\frac{c^{n+1}}{(n+1) c_{1}(M)^{n}} .
$$

Next we analyze the inner product part of $D_{Z}^{N A}\left(\mathcal{X}, \mathcal{L}_{c}\right)$ by the integral formula (3.17) for inner products.

If we have a form $\Omega \in 2 \pi c_{1}\left(\mathcal{L}_{c}\right)$ and function $\Theta$ on $\mathcal{X}$ satisfying $\iota_{Z} \Omega=i \bar{\partial} \Theta$ and $\int_{\mathcal{X}_{1}} \Theta \Omega^{n}=0$, the integral formula tells us

$$
\left\langle\alpha, \beta_{Z}\right\rangle=\frac{1}{(n+1) 2 \pi V} \int_{\mathcal{X}} \Theta \Omega^{n+1} .
$$

To construct such $\Omega$ and $\Theta$, we start with $\omega, \theta$. First pulling back by $p$, we have $\iota_{Z} p^{*} \omega=$ $i \bar{\partial} p^{*} \theta$ and $\int_{\mathcal{X}_{1}} p^{*} \theta \cdot\left(p^{*} \omega\right)^{n}=0$.

Let $s$ be a global section of $\mathcal{O}_{\mathcal{X}}(P)$ such that $(s)=P$. Take a $\beta_{Z}\left(\mathbb{S}^{1}\right)$-invariant metric $h$ on $\mathcal{O}(P)$ such that $h(s) \equiv 1$ outside of a neighborhood of $P$ such as $U_{P}:=\pi^{-1}\left\{|\tau|<\frac{1}{2}\right\}$. Denote the curvature form by

$$
-\eta:=-i \partial \bar{\partial} \log h \in 2 \pi c_{1}(\mathcal{O}(P)), \operatorname{supp} \eta \subset U_{P} .
$$

The linearization of $\beta_{Z}$ induces a Hamiltonian function $f$ satisfying $\iota_{Z} \eta=i \bar{\partial} f$. We can write down $f$ on $\mathcal{X} \backslash P$. Since $\eta=i \partial \bar{\partial} \log h(s)$ on $\mathcal{X} \backslash P$, taking $\iota_{Z}$, we have $i \bar{\partial} f=$ 
$i \bar{\partial}(Z(\log h(s)))$. It is easy to see $\log h(s)$ is $\beta_{Z}\left(\mathbb{S}^{1}\right)$-invariant, thus $Z(\log h(s))$ is real. It follows that

$$
f=Z(\log h(s))+\text { const, on } \mathcal{X} \backslash P \text {. }
$$

Then we modify $f$ by a constant such that $f=Z(\log h(s))$ on $\mathcal{X} \backslash P$. Since our choice of $h, f \equiv 0$ outside $U_{P}$.

Now we let $\Omega:=p^{*} \omega+c \eta \in 2 \pi c_{1}\left(\mathcal{L}_{c}\right)$ and $\Theta:=p^{*} \theta+c f$, they satisfy

$$
\iota_{Z} \Omega=i \bar{\partial} \Theta, \int_{\mathcal{X}_{1}} \Theta \Omega^{n}=0 .
$$

The second identity since on $\mathcal{X}_{1}$ we have $f \equiv 0, \eta \equiv 0$ and $\int_{M} \theta \omega^{n}=0$.

Putting these into integral formula (3.17), and rewrite the integrand in terms of equivariant curvatures, e.g. $\Theta+\Omega, f+\eta$. Then expand it in $c$, we obtain

$$
\begin{aligned}
\left\langle\alpha, \beta_{Z}\right\rangle & =\frac{1}{(n+1) 2 \pi V} \int_{\mathcal{X}} \Theta \Omega^{n+1}=\frac{1}{(n+1)(n+2) 2 \pi V} \int_{\mathcal{X}}(\Theta+\Omega)^{n+2} \\
& =\frac{1}{(n+1)(n+2) 2 \pi V} \sum_{i=0}^{n+2}\left(\begin{array}{c}
n+2 \\
i
\end{array}\right) \int_{\mathcal{X}}\left(p^{*} \theta+p^{*} \omega\right)^{n+2-i} \wedge(f+\eta)^{i} \cdot c^{i} .
\end{aligned}
$$

Denote $I_{i}:=\int_{\mathcal{X}}\left(p^{*} \theta+p^{*} \omega\right)^{n+2-i} \wedge(f+\eta)^{i}$. Obviously, the coefficients $I_{i}$ (of $c^{i}$ ) are independent of the choices of $\omega$ and $\eta$. We need to compute them. Note that $\left.p^{*} \omega\right|_{P} \equiv 0$ and $\left.p^{*} \theta\right|_{P} \equiv \theta\left(z_{*}\right)=\vartheta(M)$, if we can localize these integrals $I_{i}$ onto $P$, the computation would be much simpler.

Claim 6.3. For $0 \leq i \leq n$, we have $I_{i}=0$, and $I_{n+1}=-(2 \pi)^{n+1} \vartheta(M)$.

We verify this claim by localization techniques. Firstly, we see

$$
I_{0}=(n+2) \int_{\mathcal{X}} p^{*} \theta\left(p^{*} \omega\right)^{n+1}=0
$$

since $\omega^{n+1}=0$. So in the following, we assume $1 \leq i \leq n+1$.

Let $U_{\delta}=\left\{x \in \mathcal{X} \mid h(s(x))>\delta^{2}\right\}$ for small $\delta>0$, which is a tubular neighborhood of $P$. Since

$$
f+\eta=Z(\log h(s))+i \partial \bar{\partial} \log h(s)=-\left(i \bar{\partial}-\iota_{Z}\right) \partial \log h(s), \text { on } \mathcal{X} \backslash P,
$$

so in the following second row, we can replace one factor " $f+\eta$ " by $-\left(i \bar{\partial}-\iota_{Z}\right) \partial \log h(s)$.

$$
\begin{aligned}
I_{i} & =\lim _{\delta \rightarrow 0} \int_{U_{\delta}}\left(p^{*} \theta+p^{*} \omega\right)^{n+2-i} \wedge(f+\eta)^{i} \\
& =-\lim _{\delta \rightarrow 0} \int_{U_{\delta}}\left(p^{*} \theta+p^{*} \omega\right)^{n+2-i} \wedge(f+\eta)^{i-1} \wedge\left(i \bar{\partial}-\iota_{Z}\right) \partial \log h(s) \\
& =-\lim _{\delta \rightarrow 0} \int_{\partial U_{\delta}}\left(p^{*} \theta+p^{*} \omega\right)^{n+2-i} \wedge(f+\eta)^{i-1} \wedge i \partial \log h(s) .
\end{aligned}
$$

The third row is by integrating by parts (take $i \bar{\partial}-\iota_{Z}$ as a whole) and use

$$
\left(i \bar{\partial}-\iota_{Z}\right)\left(p^{*} \theta+p^{*} \omega\right)=0,\left(i \bar{\partial}-\iota_{Z}\right)(f+\eta)=0
$$


We continue to expand it, when $1<i \leq n+1$, we have

$$
\begin{aligned}
I_{i}= & -\lim _{\delta \rightarrow 0}(i-1) \int_{\partial U_{\delta}} f \cdot p^{*} \omega^{n+2-i} \wedge \eta^{i-2} \wedge i \partial \log h(s) \\
& -\lim _{\delta \rightarrow 0}(n+2-i) \int_{\partial U_{\delta}} p^{*} \theta \cdot p^{*} \omega^{n+1-i} \wedge \eta^{i-1} \wedge i \partial \log h(s)
\end{aligned}
$$

when $i=1$, we have

$$
I_{1}=-\lim _{\delta \rightarrow 0}(n+1) \int_{\partial U_{\delta}} p^{*} \theta \cdot p^{*} \omega^{n} \wedge i \partial \log h(s) .
$$

These limits will be integrals on $P$. The below lemma can be found in standard textbooks in complex geometry, e.g. [24] Page 203. We repeat the proof for completeness.

Lemma 6.4 (Localization). With the above setting and notations, let $\alpha$ be a smooth $2 n$ form, we have

$$
\lim _{\delta \rightarrow 0} \int_{\partial U_{\delta}} \alpha \wedge i \partial \log h(s)=2 \pi \int_{P} \alpha .
$$

Proof. Essentially, this is a local result. Take a local coordinates $\left\{z_{0}, \cdots, z_{n}\right\}$ and a local frame $e$ of $\mathcal{O}(P)$ around a point on $P$ such that $s=z_{0} \cdot e$. Hence $h(s)=\left|z_{0}\right|^{2} u, u:=h(e)$ is a positive smooth function. The boundary is

$$
\partial U_{\delta}=\left\{\left(z_{0}, z^{\prime}\right)|| z_{0} \mid \cdot u\left(z_{0}, z^{\prime}\right)^{1 / 2}=\delta\right\}, z^{\prime}=\left(z_{1}, \cdots, z_{n}\right) .
$$

We compute

$$
\partial \log h(s)=\frac{d z_{0}}{z_{0}}+\partial \log u
$$

Note that $\lim _{\delta \rightarrow 0} \int_{\partial U_{\delta}} \alpha \wedge i \partial \log u=0$ since $\log u$ is smooth. Thus we only consider $\int_{\partial U_{\delta}} \alpha \wedge \frac{d z_{0}}{z_{0}}$. Denote $d z^{\prime}=d z_{1} \wedge \cdots \wedge d z_{n}$, then $\alpha$ can be decomposed as

$$
\alpha=g(z) d z^{\prime} \wedge d \bar{z}^{\prime}+d z_{0} \wedge \beta+d \bar{z}_{0} \wedge \gamma,
$$

where $g$ is a function, $\beta$ and $\gamma$ are forms. It can be seen that only the first part has contribution to integral. Hence we have

$$
\begin{aligned}
\lim _{\delta \rightarrow 0} \int_{\partial U_{\delta}} \alpha \wedge i \partial \log h(s) & =\int_{P}\left(\lim _{\delta \rightarrow 0} \int g\left(z_{0}, z^{\prime}\right) \frac{i d z_{0}}{z_{0}}\right) d z^{\prime} \wedge d \bar{z}^{\prime} \\
& =2 \pi \int_{P} g\left(0, z^{\prime}\right) d z^{\prime} \wedge d \bar{z}^{\prime}=2 \pi \int_{P} \alpha
\end{aligned}
$$

Now we apply (6.3) to (6.2), note $\left.p^{*} \omega\right|_{P} \equiv 0$ and $\left.p^{*} \theta\right|_{P} \equiv \theta\left(z_{*}\right)=\vartheta(M)$,

$$
\int_{P} p^{*} \theta \cdot \eta^{n}=\vartheta(M) \cdot \int_{P} \eta^{n}=-\vartheta(M) \cdot(2 \pi)^{n}(-P)^{\cdot n+1}=(2 \pi)^{n} \vartheta(M),
$$

then Claim 6.3 follows.

We obtain an expansion

$$
\left\langle\alpha, \beta_{Z}\right\rangle=-\frac{\vartheta(M)}{(n+1) c_{1}(M)^{n}} c^{n+1}+A \cdot c^{n+2},
$$

where $A$ is a constant. Combining this with (6.1), we obtain

$$
D_{Z}^{N A}\left(\mathcal{X}, \mathcal{L}_{c}\right)=\frac{1-\vartheta(M)}{(n+1) c_{1}(M)^{n}} c^{n+1}+A \cdot c^{n+2} .
$$


If $\vartheta(M)>1, D_{Z}^{N A}\left(\mathcal{X}, \mathcal{L}_{c}\right)$ will be negative when $c \ll 1$, contradicting with relative D-semistability. Proof of Theorem 6.1 is completed.

\section{UNIFORMLY RELATIVE D-STABILITY AND REDUCED NA J-FunCTIONALS}

To define the uniform stability, we need a "norm" to measure how far an equivariant testconfiguration from a product. That is the reduced non-Archimedean J-functional introduced by Hisamoto in [22]. We restrict to the case of torus action.

7.1. Twisting a $\mathbb{C}^{*}$-equivariant test-configuration. Let $(M, L)$ be a polarized manifold. Let $T \subset \operatorname{Aut}(M, L)$ be a $m$-dimensional torus whose elements are denoted by $\sigma=\left(\sigma_{1}, \cdots, \sigma_{m}\right)$, $\sigma_{i} \in \mathbb{C}^{*}$. All the 1-parameter subgroups of $T$ constitute a lattice $\mathbb{Z}^{m}$. Specifically, each $\rho=\left(\rho_{1}, \cdots, \rho_{m}\right) \in \mathbb{Z}^{m}$ gives a subgroup

$$
\rho(\tau):=\left(\tau^{\rho_{1}}, \cdots, \tau^{\rho_{m}}\right): \mathbb{C}^{*} \rightarrow T .
$$

All the characters of $T$ constitute the dual lattice, each $\nu=\left(\nu_{1}, \cdots, \nu_{m}\right) \in \mathbb{Z}^{m}$ gives a character $\nu(\sigma):=\sigma^{\nu}=\sigma_{1}^{\nu_{1}} \cdots \sigma_{m}^{\nu_{m}}$. For any $\rho, \nu \in \mathbb{Z}^{m}$, the weight of composition $\mathbb{C}^{*} \stackrel{\rho}{\rightarrow} T \stackrel{\nu}{\rightarrow} \mathbb{C}^{*}$ is $\langle\rho, \nu\rangle=\sum_{i=1}^{m} \rho_{i} \nu_{i}$.

Definition 7.1 (twist a $T$-equivariant test-configuration). Let $(\mathcal{X}, \mathcal{L})$ be a $T$-equivariant test-configuration for $(M, L)$ with structure action $\alpha$ and fiberwise action $\beta$. Given a 1 parameter subgroup $\rho: \mathbb{C}^{*} \rightarrow T$, we modify the $\mathbb{C}^{*}$-action $\alpha$ on $\left.(\mathcal{X}, \mathcal{L})\right|_{\mathbb{C}}$ by

$$
\alpha_{\rho}(\tau):=\alpha(\tau) \circ \beta(\rho(\tau)), \tau \in \mathbb{C}^{*}
$$

and keep the other data unchanged. We denote this new test-configuration by $\left.(\mathcal{X}, \mathcal{L})\right|_{\mathbb{C}} ^{\rho}($ as a family over $\mathbb{C})$, and call it the $\rho$-twisting of $(\mathcal{X}, \mathcal{L})$.

Remark 7.2. This definition is same to [22], merely using a different formulation. Note the total space of compactification of $\left.(\mathcal{X}, \mathcal{L})\right|_{\mathbb{C}} ^{\rho}$ will be different from $\mathcal{X}$, since action $\alpha$ on the $\infty$-fiber of compactification space is always trivial. Thus if $\mathcal{X}$ dominates the trivial product $M \times \mathbb{P}^{1}\left(\alpha\right.$ only acts on $\left.\mathbb{P}^{1}\right)$, then its twisting no longer dominates trivial product. This makes it difficult to apply the intersection number formula (2.4) of $J^{N A}$ for twisted test-configurations.

Next we consider the effect of twisting on the associated filtration $\mathcal{F}(\mathcal{X}, \mathcal{L})$. By its definition (4.4), we see $F^{\mu} \mathrm{H}^{0}(k L)$ is preserved by $T$-action on $R(M, L)$. We call $\mathcal{F}(\mathcal{X}, \mathcal{L})$ is a $T$-invariant filtration. Consider the weight decomposition

$$
F^{\mu} \mathrm{H}^{0}(k L)=\bigoplus_{\nu \in \mathbb{Z}^{m}} F^{\mu} \mathrm{H}^{0}(k L)_{\nu}
$$

where $\nu$ runs over all characters of $T$. Let $\mathrm{H}^{0}(k L)_{\nu}$ be the weight- $\nu$ subspace of $\mathrm{H}^{0}(k L)$, then

$$
F^{\mu} \mathrm{H}^{0}(k L)_{\nu}:=\left\{s \in \mathrm{H}^{0}(k L)_{\nu} \mid \tau^{-\mu} \cdot \bar{s} \in \mathrm{H}^{0}\left(\left.\mathcal{X}\right|_{\mathbb{C}}, k \mathcal{L}\right)\right\} .
$$

We will denote a $T$-invariant filtration by $\left\{F^{\mu} \mathrm{H}^{0}(k L)_{\nu}\right\}$. Note that for fixed $k, \nu$, it constitutes a filtration of $\mathrm{H}^{0}(k L)_{\nu}$.

Proposition 7.3. Let $(\mathcal{X}, \mathcal{L})$ be a T-equivariant ample test-configuration with associated filtration $\mathcal{F}(\mathcal{X}, \mathcal{L})=\left\{F^{\mu} H^{0}(k L)_{\nu}\right\}$. For 1-parameter subgroup $\rho \in \mathbb{Z}^{m}$, denote by $\mathcal{F}(\mathcal{X}, \mathcal{L})^{\rho}=\left\{F_{\rho}^{\mu} H^{0}(k L)_{\nu}\right\}$ the filtration associated to twisting test-configuration $\left.(\mathcal{X}, \mathcal{L})\right|_{\mathbb{C}} ^{\rho}$. Then we have

$$
F_{\rho}^{\mu} H^{0}(k L)_{\nu}=F^{\mu-\langle\rho, \nu\rangle} H^{0}(k L)_{\nu}
$$


Proof. Let $s \in \mathrm{H}^{0}(k L)_{\nu}$, it satisfies $\sigma . s\left(\sigma^{-1} . z\right)=\sigma^{\nu} s(z)$ for $\forall \sigma \in T, z \in M$. Its equivariant extension by the new action $\alpha_{\rho}$ is

$$
\begin{aligned}
\bar{s}^{\rho}(x):=\alpha_{\rho}(\tau) s\left(\alpha_{\rho}\left(\tau^{-1}\right) x\right) & =\alpha(\tau) \beta(\rho(\tau)) s\left(\beta\left(\rho\left(\tau^{-1}\right)\right) \alpha\left(\tau^{-1}\right) x\right) \\
& =\tau^{\langle\rho, \nu\rangle} \cdot \alpha(\tau) s\left(\alpha\left(\tau^{-1}\right) x\right)=\tau^{\langle\rho, \nu\rangle} \cdot \bar{s}(x),
\end{aligned}
$$

where $x \in \mathcal{X}_{\tau}, \alpha_{\rho}\left(\tau^{-1}\right) x \in \mathcal{X}_{1} \cong M$, the restriction of $\beta$ on $\left(\mathcal{X}_{1}, \mathcal{L}_{1}\right)$ is the given $T$ action. Then the formula follows by (7.1).

By taking base change, twisting operation can be extended to all rational $\rho$. For irrational $\rho \in \mathbb{R}^{m}$, we can define $\rho$-twisting of the associated filtration by the formula in above proposition.

Definition 7.4 (twist the associated filtration). Let $(\mathcal{X}, \mathcal{L})$ be a T-equivariant ample testconfiguration. Its associated $T$-invariant filtration is $\mathcal{F}(\mathcal{X}, \mathcal{L})=\left\{F^{t} H^{0}(k L)_{\nu}\right\}$. For any $\rho \in \mathbb{R}^{m}$, we define a new $T$-invariant filtration (called the $\rho$-twisting of $\mathcal{F}(\mathcal{X}, \mathcal{L})$ ) denoted by $\mathcal{F}(\mathcal{X}, \mathcal{L})^{\rho}=\left\{F_{\rho}^{t} H^{0}(k L)_{\nu}\right\}$,

$$
F_{\rho}^{t} H^{0}(k L)_{\nu}:=F^{t-\langle\rho, \nu\rangle} H^{0}(k L)_{\nu}, F_{\rho}^{t} H^{0}(k L):=\bigoplus_{\nu \in \mathbb{Z}^{m}} F_{\rho}^{t} H^{0}(k L)_{\nu} .
$$

Recall that $F^{t} H^{0}(k L)=F^{\lceil t\rceil} H^{0}(k L)$ for all $t \in \mathbb{R}$.

7.2. Uniformly relative D-stability. For a probability measure $\mathfrak{m}$ on $\mathbb{R}$ with bounded support, we set

$$
j(\mathfrak{m}):=\sup \operatorname{suppm}-\int_{\mathbb{R}} \lambda d \mathfrak{m} \geq 0 \text {. }
$$

Definition 7.5 (reduced NA J-functionals). (1) Let $\mathcal{F}$ be an admissible filtration of the section ring $R(M, L)$. We define

$$
J^{N A}(\mathcal{F}):=j(L M(\mathcal{F})),
$$

where $\operatorname{LM}(\mathcal{F})$ is the limit measure of $\mathcal{F}$, see Theorem 4.7. In particular, take $\mathcal{F}$ to be the associated filtration $\mathcal{F}(\mathcal{X}, \mathcal{L})$ to an ample test-configuration $(\mathcal{X}, \mathcal{L})$ for $(M, L)$, it gives $J^{N A}(\mathcal{X}, \mathcal{L})(2.3)$

(2) Let $(\mathcal{X}, \mathcal{L})$ be a $T$-equivariant ample test-configuration for $(M, L)$. The reduced (means modulo T-action) NA J-functional is defined by

$$
J_{T}^{N A}(\mathcal{X}, \mathcal{L}):=\inf _{\rho \in \mathbb{R}^{m}} J^{N A}\left(\mathcal{F}(\mathcal{X}, \mathcal{L})^{\rho}\right),
$$

where $\mathcal{F}(\mathcal{X}, \mathcal{L})^{\rho}$ is $\rho$-twisting of $\mathcal{F}(\mathcal{X}, \mathcal{L})(7.2)$.

By [7] Corollary $\mathrm{B}, J^{N A}(\mathcal{X}, \mathcal{L})$ is zero if and only if $(\mathcal{X}, \mathcal{L})$ is trivial product $M \times \mathbb{P}^{1}$ (i.e. action $\alpha$ is trivial). Roughly speaking, $J_{T}^{N A}$ measures how far is $(\mathcal{X}, \mathcal{L})$ from product test-configurations (may have a nontrivial action $\alpha$ via $T$-action).

Definition 7.6. With the same assumptions in Definition 5.10, here we take G is torus T. $M$ is said to be uniformly $D$-stable relative to $T$ if there exists a $\delta>0$ such that

$$
D_{Z}^{N A}(\mathcal{X}, \mathcal{L}) \geq \delta \cdot J_{T}^{N A}(\mathcal{X}, \mathcal{L})
$$

for all T-equivariant ample test-configuration for $\left(M,-K_{M}\right)$.

We want to show uniformly relative D-stability implies $\vartheta(M)<1$. Continue the proof for semistability implies $\vartheta(M) \leq 1$, next we need to expand $J_{T}^{N A}\left(\mathcal{X}, \mathcal{L}_{c}\right)$ in parameter $c$. As noted in Remark 7.2, the intersection number formula of $J^{N A}$ is difficult to use when 
we make twisting. Hence in the sequel, we give a convex-geometry description for $J_{T}^{N A}$ via Okounkov bodies. Finally, we will see $J_{T}^{N A}\left(\mathcal{X}, \mathcal{L}_{c}\right)$ is attained by the trivial twisting (i.e. $\rho=0$ ) when $c \ll 1$, then we are done.

7.3. Infinitesimal Okounkov bodies. In [35], Okounkov associated a convex body $\triangle \subset$ $\mathbb{R}^{n}$ to a polarized algebraic variety $(X, L)$ (only need $L$ is big), its Euclidean volume gives the volume of $L$. Boucksom-Chen [6] showed an admissible filtration $\mathcal{F}$ of $R(X, L)$ gives rise to a concave function $G[\mathcal{F}]$ on $\triangle$, called the concave transform of $\mathcal{F}$, and the pushforward of Lebesgue measure by $G[\mathcal{F}]$ gives the limit measure $\operatorname{LM}(\mathcal{F})$. In particular, take $\mathcal{F}=\mathcal{F}(\mathcal{X}, \mathcal{L})$ for a test-configuration $(\mathcal{X}, \mathcal{L})$, the associated concave transform $G[\mathcal{X}, \mathcal{L}]$ encodes many information of $(\mathcal{X}, \mathcal{L})$, such as $\operatorname{DH}(\mathcal{X}, \mathcal{L}), J^{N A}(\mathcal{X}, \mathcal{L})$.

In Okounkov's construction, we need to choose a flag of subvarieties in $M$, the resulting $\triangle$ depends heavily on the choices. In our setting, we need a flag adapting to the $T$-action. For this, a local version called infinitesimal Okounkov body introduced in [26] is more suitable for our setting. Its construction is as following.

Let $(M, L)$ be a polarized manifold with a lifted $m$-dimensional torus action $\beta: T \rightarrow$ $\operatorname{Aut}(M, L)$. By [1], there exists a $T$-fixed point $z_{*} \in M$. Let $V_{\bullet}$ be a full flag of subspaces in the tangent space $T_{z_{*}} M$ (called an infinitesimal flag at $z_{*}$ ). Let

$$
q: \hat{M} \rightarrow M
$$

be the blowup at $z_{*}$. Then $V_{\bullet}$ induces a flag of subvarieties $Y_{\bullet}$ in $\hat{M}$ by taking projectivization of each subspace in $V_{\bullet}$ (subvarieties in $Y_{\bullet}$ are contained in the exceptional divisor). Then the infinitesimal Okounkov body is the Okounkov body associated to datum $\left(\hat{M}, q^{*} L, Y_{\bullet}\right)$. More specifically, note $q^{*} L$ is big, and the pullback

$$
q^{*}: \mathrm{H}^{0}(M, k L) \rightarrow \mathrm{H}^{0}\left(\hat{M}, k q^{*} L\right)
$$

is an isomorphism $\left(q_{*} \mathcal{O}_{\hat{M}}=\mathcal{O}_{M}\right)$. For $s \in \mathrm{H}^{0}(M, k L)$, the vanishing orders of $q^{*} s$ along $Y_{\bullet}$ give us a rank- $n$ valuation $\mathcal{V}(s)$, then $\triangle$ will be defined by these values.

Next we go through the construction in details. First we choose an infinitesimal flag $V$. adapted to $T$-action.

Step 1: take a $\beta$-invariant local frame $e$ around $z_{*}$.

Suppose $\beta$ acts on $L_{z_{*}}$ via character $\nu_{0}^{*} \in \mathbb{Z}^{m}$. Since $L$ is ample, there exists $\kappa_{0} \in \mathbb{N}$ and $s \in \mathrm{H}^{0}\left(M, \kappa_{0} L\right)$ such that $s\left(z_{*}\right) \neq 0$. Decompose $s=\sum_{\nu} s_{\nu}$ w.r.t. action $\beta$, where $s_{\nu} \in \mathrm{H}^{0}\left(M, \kappa_{0} L\right)_{\nu}$ satisfying

$$
\sigma . s_{\nu}\left(\sigma^{-1} . z\right)=\sigma^{\nu} \cdot s_{\nu}(z), \text { for } \sigma \in T, z \in M .
$$

Take $z=z_{*}$ we see $s_{\nu}\left(z_{*}\right) \neq 0$ only if $\nu=\nu_{0}^{*}$. Now we set

$$
e:=s_{\nu_{0}^{*}}
$$

which is a $\beta$-invariant local frame of $L^{\kappa_{0}}$ on the $\beta$-invariant open set $\left\{s_{\nu_{0}^{*}} \neq 0\right\} \ni z_{*}$.

Step 2: take a local coordinates $\left(z_{i}\right)_{i=1}^{n}$ centered at $z_{*}\left(\right.$ i.e. $z_{i}\left(z_{*}\right)=0$ ) such that action $\beta$ is diagonal under $\left(z_{i}\right)$ up to higher order terms.

which diagonalize s action $\beta$ at first order.

Since $\beta$ induces a $T$-action on $T_{z_{*}}^{(1,0)} M$, let $\nu_{1}^{*}, \cdots, \nu_{n}^{*} \in \mathbb{Z}^{m}$ be the associated characters. By a linear transformation, we can find a coordinates $\left(z_{i}\right)$ centered at $z_{*}$ such that

$$
z_{i}(\beta(\sigma) . z)=\nu_{i}^{*}(\sigma) \cdot z_{i}+\text { higher order terms, for } \forall \sigma \in T, 1 \leq i \leq n .
$$

Where "higher order terms" means monomials of $\left(z_{i}\right)$ with order $\geq 2$.

Step 3: choose an infinitesimal flag $V_{\bullet}$. 
We describe the blowup $q: \hat{M} \rightarrow M$ explicitly by coordinates. Let $E=\mathbb{P}\left(T_{z_{*}} M\right)$ be the exceptional divisor. Near $E$, using coordinate $\left(z_{i}\right), \hat{M}$ is given by

$$
\left\{\left(\left(z_{i}\right),\left[w_{1}, \cdots, w_{n}\right]\right) \in M \times \mathbb{P}^{n-1} \mid z_{i} w_{j}=z_{j} w_{i}, \forall i, j\right\} .
$$

On the open set $\left\{w_{1} \neq 0\right\}$, we define $u_{1}=z_{1}, u_{i}=w_{i} / w_{1}$ for $2 \leq i \leq n$, then $\left(u_{i}\right)_{i=1}^{n}$ constitutes a local coordinates on $\hat{M}$ around $E$. Under this coordinates, $q$ is given by

$$
q:\left(u_{1}, \cdots, u_{n}\right) \longmapsto\left(z_{i}\right)=\left(u_{1}, u_{1} u_{2}, \cdots, u_{1} u_{n}\right) .
$$

Now we choose a full flag of subspaces in $T_{z_{*}} M$,

$$
V_{\bullet}: T_{z_{*}} M \supset \operatorname{span}\left\{\partial_{1}, \partial_{3}, \cdots, \partial_{n}\right\} \supset \operatorname{span}\left\{\partial_{1}, \partial_{4}, \cdots, \partial_{n}\right\} \supset \cdots \supset \operatorname{span}\left\{\partial_{1}\right\}
$$

where $\partial_{i}:=\frac{\partial}{\partial z_{i}}$. Taking projectivization, it induces a flag of subvarieties in $\hat{M}$,

$$
\begin{aligned}
Y_{\bullet}: & Y_{1}=E=\overline{\left\{u_{1}=0\right\}} \supset Y_{2}=\overline{\left\{u_{1}=u_{2}=0\right\}} \supset \cdots \\
& \cdots \supset Y_{n}=\left\{u_{1}=\cdots=u_{n}=0\right\} .
\end{aligned}
$$

Step 4: define valuation $\mathcal{V}$.

Take a nonzero $s \in \mathrm{H}^{0}(M, k L)$, then $s^{\kappa_{0}} \in \mathrm{H}^{0}\left(M, \kappa_{0} k L\right)$ (see Step 1 for $\left.\kappa_{0}\right)$, express $s^{\kappa_{0}}=f(z) \cdot e^{k}$ in a neighborhood of $z_{*}$. We expand $f$ as power series,

$$
f(z)=\sum_{a \geq 0} c_{a} \cdot z^{a}, a=\left(a_{1}, \cdots, a_{n}\right), z^{a}=z_{1}^{a_{1}} \cdots z_{n}^{a_{n}} .
$$

Pulling back by $q$, we have $q^{*} s^{\kappa_{0}}=(f \circ q) q^{*} e^{k}$. By (7.6), we have

$$
(f \circ q)(u)=\sum_{a \geq 0} c_{a} \cdot u_{1}^{\sum a_{i}} u_{2}^{a_{2}} \cdots u_{n}^{a_{n}} .
$$

The vanishing orders of $f \circ q$ along the subvarieties in flag (7.8) define a rank- $n$ valuation

$$
\mathcal{V}(s):=\frac{1}{\kappa_{0}} \min \left\{\left(\sum_{i=1}^{n} a_{i}, a_{2} \cdots, a_{n}\right) \mid c_{a} \neq 0\right\} \in \frac{1}{\kappa_{0}} \mathbb{N}^{n}, \mathcal{V}(0):=\infty,
$$

where the minimizer is taken w.r.t. the lexicographic order. It satisfies

$$
\begin{gathered}
\mathcal{V}(s \otimes t)=\mathcal{V}(s)+\mathcal{V}(t), \text { for } s \in \mathrm{H}^{0}(k L), t \in \mathrm{H}^{0}(l L) ; \\
\mathcal{V}(s+t) \geq \min \{\mathcal{V}(s), \mathcal{V}(t)\}, \text { for } s, t \in \mathrm{H}^{0}(k L),
\end{gathered}
$$

where $\geq$ is the lexicographic order.

Remark 7.7. Note the first component of $\mathcal{V}(s)$ is the multiplicity $\operatorname{ord}_{z_{*}}(s)=\operatorname{ord}_{E}\left(q^{*} s\right)$. In the definition of $\mathcal{V}(s)$, the order we used takes the multiplicity $\sum_{i=1}^{n} a_{i}$ as priority, contrasting with the lexicographic order on $\left(a_{1}, \cdots, a_{n}\right)$. We choose this order because it is compatible with expansion of power series, this is required for Proposition 7.10 in the sequel. This is also the reason why we chose infinitesimal flag $V_{\bullet}$ (7.7).

Step 5: define the (infinitesimal) Okounkov body $\triangle$.

Collecting all the values of $\mathcal{V}(s)$, we obtain a semigroup

$$
\Gamma(L):=\left\{(\mathcal{V}(s), k) \mid 0 \neq s \in \mathrm{H}^{0}(M, k L), k \in \mathbb{N}\right\} \subset \frac{1}{\kappa_{0}} \mathbb{N}^{n+1} .
$$

Let $\Sigma(L) \subset \mathbb{R}^{n+1}$ be the convex cone spanned by $\Gamma(L)$. 
Definition 7.8 (infinitesimal Okounkov body). The infinitesimal Okounkov body associated to the infinitesimal flag $V_{\bullet}(7.7)$ at the $T$-fixed point $z_{*}$ is defined by

$$
\triangle(L):=\{x \mid(x, 1) \in \Sigma(L)\} \subset \mathbb{R}^{n} .
$$

It is the ordinary Okounkov body of $q^{*} L$ associated to the flag of subvarieties (7.8) in $\hat{M}$.

Remark 7.9. $\triangle(L)$ only depends on the infinitesimal flag (7.7), but this flag depends on the action $\beta$ around $z_{*}$. Hence $\triangle(L)$ depends on action $\beta$.

On the other hand, $\triangle(L)$ also can be defined as closure

$$
\triangle(L)=\overline{\bigcup_{k \geq 1} \triangle_{k}(L)}, \triangle_{k}(L):=\left\{\mathcal{V}(s) / k \mid 0 \neq s \in \mathrm{H}^{0}(M, k L)\right\} .
$$

Okounkov body $\triangle(L)$ encodes the volume of $L$. By [26] Theorem A, we have

$$
|\triangle(L)|=\frac{\left(q^{*} L\right)^{n}}{n !}=\frac{L^{n}}{n !}
$$

where $|\cdot|$ is the standard Lebesgue measure.

In the following, for an eigen-section $s \in \mathrm{H}^{0}(M, k L)_{\nu}$, we see its weight $\nu \in \mathbb{Z}^{m}$ can be linearly determined by its valuation $\mathcal{V}(s)$.

Proposition 7.10 (weight v.s. valuation). For any weight $\nu \in \mathbb{Z}^{m}$ and nonzero $s \in$ $H^{0}(M, k L)_{\nu}$. Suppose $\mathcal{V}(s) / k=\left(x_{1}, \cdots, x_{n}\right)$, then we have

$$
\frac{\nu}{k}=-x_{1} \nu_{1}^{*}+\sum_{i=2}^{n} x_{i}\left(\nu_{1}^{*}-\nu_{i}^{*}\right)+\frac{\nu_{0}^{*}}{\kappa_{0}}=: H_{\beta}(x) .
$$

See above Step 1-2 for constants $\kappa_{0}, \nu_{0}^{*}, \cdots, \nu_{n}^{*}$, which depend on action $\beta$ around $z_{*}$. In particular, the weight $\nu$ of $s$ is an affine function $H_{\beta}$ of the valuation $\mathcal{V}(s)$.

Proof. By the local frame $e$ and local coordinates $\left(z_{i}\right)$ near $z_{*}$ in Step 1-2, assume $s^{\kappa_{0}}=$ $f(z) \cdot e^{k}$ and expand $f=\sum_{a \geq 0} c_{a} \cdot z^{a}$ near $z_{*}$. Since $s$ and $e$ satisfy equivariant condition, i.e.

$$
\sigma . s\left(\sigma^{-1} . z\right)=\sigma^{\nu} s(z), \sigma . e\left(\sigma^{-1} . z\right)=\sigma^{\nu_{0}^{*}} e(z), \text { for } \forall \sigma \in T .
$$

It implies

$$
f(\sigma . z)=\sigma^{k \nu_{0}^{*}-\kappa_{0} \nu} f(z), \text { for } \forall \sigma \in T .
$$

Let $z^{a}=z_{1}^{a_{1}} \cdots z_{n}^{a_{n}}$ be the minimum monomial in the expansion of $f$ w.r.t. the lexicographic order of $\left(\sum_{i=1}^{n} a_{i}, a_{2} \cdots, a_{n}\right)$. By the definition (7.10) of $\mathcal{V}(s)$, we have

$$
\left(x_{1}, \cdots, x_{n}\right)=\frac{1}{\kappa_{0} k}\left(\sum_{i=1}^{n} a_{i}, a_{2} \cdots, a_{n}\right) .
$$

Put the expansion (7.5) of action $\beta$ into (7.12), we obtain

$$
c_{a} \cdot \sigma^{a \cdot \nu^{*}} z^{a}+\cdots=c_{a} \cdot \sigma^{k \nu_{0}^{*}-\kappa_{0} \nu} z^{a}+\cdots, \forall \sigma \in T
$$

where $a \cdot \nu^{*}:=\sum_{i=1}^{n} a_{i} \nu_{i}^{*}$. It follows that $\sum_{i=1}^{n} a_{i} \nu_{i}^{*}=k \nu_{0}^{*}-\kappa_{0} \nu$. Then replace $\left(a_{i}\right)$ by $\left(x_{i}\right)$, we obtain $(7.11)$. 
7.4. Concave transforms of filtrations. It is showed in [6], an admissible filtration of section ring gives rise to a concave function on the Okounkov body. In our definition, $\triangle(L)$ is the Okounkov body of $q^{*} L$. Since

$$
q^{*}: R(M, L) \rightarrow R\left(\hat{M}, q^{*} L\right)
$$

is an isomorphism, an admissible filtration of $R(M, L)$ induces another one for $R\left(\hat{M}, q^{*} L\right)$, thus by [6] it gives rise to a concave function on $\triangle(L)$. Next we review the construction in [6].

Let $\mathcal{F}=\left\{F^{t} \mathrm{H}^{0}(k L)\right\}$ be an admissible filtration of $R(M, L)$. Let

$$
\triangle_{k}^{t}(L, \mathcal{F}):=\left\{\mathcal{V}(s) / k \mid 0 \neq s \in F^{t} \mathrm{H}^{0}(k L)\right\} \subset \triangle_{k}(L),
$$

then $\left\{\triangle_{k}^{t}(L, \mathcal{F})\right\}_{t}$ constitutes a filtration of subsets of $\triangle_{k}(L)$, which is decreasing in $t$ and becoming empty when $t>C k$ for a constant $C>0$. We also have (see [26] Lemma 1.4)

$$
\left|\triangle_{k}^{t}(L, \mathcal{F})\right|=\operatorname{dim} F^{t} \mathrm{H}^{0}(k L) .
$$

For each $k \geq 1$, we define a function $G_{k}$ on $\triangle_{k}(L)$ (called $k$-th approximation function),

$$
G_{k}(x):=\sup \left\{t / k \mid x \in \triangle_{k}^{t}(L, \mathcal{F})\right\}, \text { for } x \in \triangle_{k}(L) .
$$

With these $G_{k}$, we define a function on $\bigcup_{k \geq 1} \triangle_{k}(L)$,

$$
G[\mathcal{F}](x):=\sup \left\{G_{k}(x) \mid k \text { s.t. } x \in \triangle_{k}(L)\right\}, \text { for } x \in \bigcup_{k \geq 1} \triangle_{k}(L) .
$$

Theorem 7.11 (concave transform [6]). Let $\mathcal{F}$ be an admissible filtration of the section ring $R(M, L)$. The function $G[\mathcal{F}]$ defined above can be extended to a bounded concave function on the interior of $\triangle(L)$ (called the concave transform of $\mathcal{F}$ ). Moreover, the pushforward

$$
G[\mathcal{F}]_{\#}\left(\frac{d x}{|\triangle(L)|}\right)
$$

of the normalized Lebesgue measure is the limit measure $\operatorname{LM}(\mathcal{F})$.

By this theorem, we can use $G[\mathcal{F}]$ to express $J^{N A}$,

$$
J^{N A}(\mathcal{F})=j(\operatorname{LM}(\mathcal{F}))=\sup _{\triangle(L)} G[\mathcal{F}]-\int_{\triangle(L)} G[\mathcal{F}] \frac{d x}{|\triangle(L)|} .
$$

7.5. A convex-geometry description for $J_{T}^{N A}$. Let $(\mathcal{X}, \mathcal{L})$ be a $T$-equivariant ample testconfiguration for $(M, L)$. In order to express $J_{T}^{N A}(\mathcal{X}, \mathcal{L})$ via (7.14), we consider the concave transform of twisted filtration $\mathcal{F}(\mathcal{X}, \mathcal{L})^{\rho}$.

For each $\nu \in \mathbb{Z}^{m}$, let

$$
\triangle_{k}(L)_{\nu}:=\left\{\mathcal{V}(s) / k \mid 0 \neq s \in \mathrm{H}^{0}(M, k L)_{\nu}\right\} .
$$

Proposition 7.10 ensures $\triangle_{k}(L)_{\nu}$ are contained in different hyperplanes for different $\nu$, hence we have disjoint union

$$
\triangle_{k}(L)=\bigsqcup_{\nu \in \mathbb{Z}^{m}} \triangle_{k}(L)_{\nu}
$$

Let $\mathcal{F}(\mathcal{X}, \mathcal{L})=\left\{F^{t} \mathrm{H}^{0}(k L)_{\nu}\right\}$ be the associated $T$-invariant filtration to $(\mathcal{X}, \mathcal{L})$. Similarly, for each $\nu \in \mathbb{Z}^{m}$, let

$$
\triangle_{k}^{t}(L, \mathcal{F})_{\nu}:=\left\{\mathcal{V}(s) / k \mid 0 \neq s \in F^{t} \mathrm{H}^{0}(k L)_{\nu}\right\} \subseteq \triangle_{k}(L)_{\nu}
$$


Then $\left\{\triangle_{k}^{t}(L, \mathcal{F})_{\nu}\right\}_{t}$ constitutes a filtration of subsets of $\triangle_{k}(L)_{\nu}$ and we have disjoint union

$$
\triangle_{k}^{t}(L, \mathcal{F})=\bigsqcup_{\nu \in \mathbb{Z}^{m}} \triangle_{k}^{t}(L, \mathcal{F})_{\nu}
$$

By the definition of $k$-th approximation function $G_{k}$, for $x \in \triangle_{k}(L)_{\nu}$, we have

$$
G_{k}(x)=\sup \left\{t / k \mid x \in \triangle_{k}^{t}(L, \mathcal{F})_{\nu}\right\} .
$$

Proof of Theorem 1.4. Denote by $G_{k}^{\rho}$ be the $k$-th approximation function associated to twisted filtration $\mathcal{F}^{\rho}:=\mathcal{F}(\mathcal{X}, \mathcal{L})^{\rho}$, i.e. for $x \in \triangle_{k}(L)_{\nu}$, we define

$$
G_{k}^{\rho}(x)=\sup \left\{t / k \mid x \in \triangle_{k}^{t}\left(L, \mathcal{F}^{\rho}\right)_{\nu}\right\}
$$

By the definition of $\rho$-twisting (7.2), we have

$$
G_{k}^{\rho}(x)=\sup \left\{s / k \mid x \in \triangle_{k}^{s}(L, \mathcal{F})_{\nu}\right\}+\frac{\langle\rho, \nu\rangle}{k}=G_{k}(x)+\left\langle\rho, \frac{\nu}{k}\right\rangle .
$$

We can express $\left\langle\rho, \frac{\nu}{k}\right\rangle$ by $x$. In fact, since $x \in \triangle_{k}(L)_{\nu}$, suppose $x=\mathcal{V}(s) / k$ for a nonzero $s \in \mathrm{H}^{0}(M, k L)_{\nu}$. By the relation (7.11), we have

$$
\left\langle\rho, \frac{\nu}{k}\right\rangle=\left\langle\rho, H_{\beta}(x)\right\rangle=-\left\langle\rho, \nu_{1}^{*}\right\rangle x_{1}+\sum_{i=2}^{n}\left\langle\rho, \nu_{1}^{*}-\nu_{i}^{*}\right\rangle x_{i}+\frac{1}{\kappa_{0}}\left\langle\rho, \nu_{0}^{*}\right\rangle .
$$

The RHS is an affine function of $x$ with coefficients independent of $k$. By the definition of concave transforms, we obtain $G\left[\mathcal{F}^{\rho}\right]=G+\left\langle\rho, H_{\beta}\right\rangle$. Put this into (7.14), we obtain (1.7). The part (2) of this theorem is obvious.

Remark 7.12. By (1.7), we see $J^{N A}\left(\mathcal{F}(\mathcal{X}, \mathcal{L})^{\rho}\right)$ is proper as a function of $\rho \in \mathbb{R}^{m}$, thus the infimum $J_{T}^{N A}$ can be attained by some $\rho_{0} \in \mathbb{R}^{m}$, but $\rho_{0}$ may be irrational.

\section{UNIFORMLY RELATIVE D-STABILITY IMPLIES $\vartheta(M)<1$}

Now we can complete the proof of our main theorem. The concave transform associated to the deformation to normal cone had been considered in [41].

Theorem 8.1. Let $M$ be a Fano manifold, $L=-K_{M}$. Let $T \subset A u t^{0}(M)$ be a torus. If $M$ is uniformly $D$-stable relative to $T$ in the sense of Definition 7.6, then $\vartheta(M) \leq 1-\delta$.

Proof. We take the canonical lifting of $T$-action $\beta: T \rightarrow \operatorname{Aut}\left(M,-K_{M}\right)$. Let $z_{*}$ be the $T$-fixed point provided by Proposition 6.2, and $\triangle(L)$ is the infinitesimal Okounkov body associated to $z_{*}$ and infinitesimal flag $V_{\bullet}(7.7)$. Let $\left\{\left(\mathcal{X}, \mathcal{L}_{c}\right) \mid \mathbb{Q}_{+} \ni c \ll 1\right\}$ be the family of $T$-equivariant test-configurations constructed in the proof of Theorem 6.1.

Let $\mathcal{F}_{c}=\left\{F_{c}^{t} \mathrm{H}^{0}(k L)\right\}$ be the filtration associated to $\left(\mathcal{X}, \mathcal{L}_{c}\right)$ (where subscript " $c$ " does not mean twisting!). The Lemma 5.17 in [7] tells us

$$
F_{c}^{t} \mathrm{H}^{0}(k L)=\left\{s \in \mathrm{H}^{0}(k L) \mid \operatorname{ord}_{z_{*}}(s) \geq t+c k\right\}, \text { when } t \leq 0 ;
$$

and $F_{c}^{t} \mathrm{H}^{0}(k L)=0$ when $t>0$. Let $q: \hat{M} \rightarrow M$ be the blowup at $z_{*}$ with exceptional divisor $E$, we have

$$
F_{c}^{t} \mathrm{H}^{0}(k L) \cong \mathrm{H}^{0}\left(\hat{M}, k q^{*} L-\lceil t+c k\rceil E\right), \text { when }-c k \leq t \leq 0 ;
$$

$F_{c}^{t} \mathrm{H}^{0}(k L) \cong \mathrm{H}^{0}\left(\hat{M}, k q^{*} L\right)$ when $t<-c k$; and $F_{c}^{t} \mathrm{H}^{0}(k L)=0$ when $t>0$.

From this we obtain the DH measure (see Proposition 8.5 [7]),

$$
\operatorname{DH}\left(\mathcal{X}, \mathcal{L}_{c}\right)=\frac{n}{L^{n}}(\lambda+c)^{n-1} \mathbf{1}_{[-c, 0]} d \lambda+\left(1-\frac{c^{n}}{L^{n}}\right) \delta_{0},
$$


where $\mathbf{1}_{[-c, 0]}$ is characteristic function, $d \lambda$ is Lebesgue measure and $\delta_{0}$ is the Dirac measure at 0 . It follows that

$$
J^{N A}\left(\mathcal{X}, \mathcal{L}_{c}\right)=j\left(\operatorname{DH}\left(\mathcal{X}, \mathcal{L}_{c}\right)\right)=\frac{c^{n+1}}{(n+1) L^{n}} .
$$

Next we consider the concave transform of $\mathcal{F}_{c}$. Since the first component of $\mathcal{V}(s)$ is $\operatorname{ord}_{z_{*}}(s)=\operatorname{ord}_{E}\left(q^{*} s\right)$, thus when $t \leq 0$ we have

$$
\triangle_{k}^{t}\left(L, \mathcal{F}_{c}\right)=\left\{\mathcal{V}(s) / k \mid 0 \neq s \in F_{c}^{t} \mathrm{H}^{0}(k L)\right\}=\triangle_{k}(L) \cap\left\{x_{1} \geq \frac{t}{k}+c\right\} ;
$$

and $\triangle_{k, t}\left(L, \mathcal{F}_{c}\right)=\emptyset$ when $t>0$. It follows that for $x \in \triangle_{k}(L)$,

$$
G_{k}(x)=\sup \left\{t / k \mid x \in \triangle_{k}^{t}\left(L, \mathcal{F}_{c}\right)\right\}=\min \left\{x_{1}-c, 0\right\} .
$$

Hence the concave transform is

$$
G\left[\mathcal{F}_{c}\right]=\min \left\{x_{1}-c, 0\right\} .
$$

Next we consider $J_{T}^{N A}\left(\mathcal{X}, \mathcal{L}_{c}\right)$, denote by $\mathcal{F}_{c}^{\rho}$ the $\rho$-twisting of $\mathcal{F}_{c}$. We claim the infimum

$$
J_{T}^{N A}\left(\mathcal{X}, \mathcal{L}_{c}\right):=\inf _{\rho \in \mathbb{R}^{m}} J^{N A}\left(\mathcal{F}_{c}^{\rho}\right)
$$

is attained at $\rho=0$ when $c \ll 1$. First we note the following facts:

(1) $\triangle(L)$ is a convex body with nonempty interior, and by the definition of $\mathcal{V}(7.10)$ it is contained in region $\left\{x \in \mathbb{R}_{\geq}^{n} \mid x_{1} \geq x_{2}+\cdots+x_{n}\right\}$.

(2) $\inf _{\triangle} x_{1}=0$. If not, $\triangle \subset\left\{x_{1} \geq c\right\}$ for some $1 \gg c \in \mathbb{Q}_{+}$, thus $G\left[\mathcal{F}_{c}\right] \equiv 0$ on $\triangle$. However, since $\operatorname{DH}\left(\mathcal{X}, \mathcal{L}_{c}\right)$ is the pushforward of Lebesgue measure by $G\left[\mathcal{F}_{c}\right]$, this contradicts with (8.1). Then combine inf $\triangle x_{1}=0$ with (1), we know $0 \in \triangle(L)$.

With these facts, the claim follows from the convex-geometry description of $J_{T}^{N A}$, see Theorem 1.4 (2) and refer to Figure 1.1.

Hence we have

$$
J_{T}^{N A}\left(\mathcal{X}, \mathcal{L}_{c}\right)=J^{N A}\left(\mathcal{X}, \mathcal{L}_{c}\right)=\frac{c^{n+1}}{(n+1) L^{n}}, \text { when } c \ll 1 .
$$

Combining this with the expansion of Berman-Ding invariant (6.4), thus the uniform stability condition (7.4) implies $\vartheta(M) \leq 1-\delta$.

Acknowledgments: The author would like to thank Feng Wang, Jia-xiang Wang and Naoto Yotsutani for helpful discussions, and specially thank Mingchen Xia for valuable revision suggestions. The author is supported by Grants: National Natural Science Foundation of China (NSFC) (No. 751203123) and Fundamental Research Funds for the Central Universities (No. 531118010149).

\section{REFERENCES}

[1] Atiyah, M.: Convexity and commuting Hamiltonians. Bull. Lond. Math. Soc. 14(1), 1-15 (1982)

[2] Apostolov, V., Calderbank, D., Gauduchon, P., Tønnesen-Friedman, C.: Hamiltonian 2-forms in Kähler geometry, III Extremal metrics and stability. Invent. Math. 173(3), 547-601 (2008)

[3] Berman, R.: K-polystability of Q-Fano varieties admitting Kähler-Einstein metrics. Invent. Math. 203(3), 973-1025 (2016)

[4] Berman, R., Boucksom, S., Jonsson, M.: A variational approach to the Yau-Tian-Donaldson conjecture. To appear in J. Amer. Math. Soc. arXiv:1509.04561v3

[5] Berman, R., Witt Nyström, D.: Complex optimal transport and the pluripotential theory of Kähler-Ricci solitons. arXiv: 1401.8264

[6] Boucksom, S., Chen, H.: Okounkov bodies of filtered linear series. Compos. Math. 147(4), 1205-1229 (2011) 
[7] Boucksom, S., Hisamoto, T., Jonsson. M.: Uniform K-stability, Duistermaat-Heckman measures and singularities of pairs. Ann. Inst. Fourier (Grenoble). 67(2), 743-841 (2017)

[8] Boucksom, S., Hisamoto, T., Jonsson. M.: Uniform K-stability and asymptotics of energy functionals in Kähler geometry. J. Eur. Math. Soc. 21(9), 2905-2944 (2019)

[9] Chen, X., Cheng, J.: On the constant scalar curvature Kähler metrics, general automorphism group. arXiv: 1801.05907

[10] Chu, J., Tosatti, V., Weinkove, B.: C1,1 regularity for degenerate complex Monge-Ampère equations and geodesic rays. Comm. Partial Differential Equations. 43(2), 292-312 (2018)

[11] Collins, T., Hisamoto, T., Takahashi, R.: The inverse Monge-Ampère flow and applications to KählerEinstein metrics. To appear in J. Differential Geom. arXiv:1712.01685v2

[12] Dervan, R.: Relative K-stability for Kähler manifolds. Math. Ann. 372(3-4), 859-889 (2018)

[13] Donaldson, S.: Lower bounds on the Calabi functional. J. Differential Geom. 70(3), 453-472 (2005)

[14] Donaldson, S.: The Ding functional, Berndtsson convexity and moment maps. Geometry, Analysis and Probability (In Honor of Jean-Michel Bismut). Birkhäuser, Cham (2017)

[15] Futaki, A., Mabuchi, T.: Bilinear forms and extremal Kähler vector fields associated with Kähler classes. Math. Ann. 301(1), 199-210 (1995)

[16] Han, J., Li, C.: On the Yau-Tian-Donaldson conjecture for generalized Kähler-Ricci soliton equations. arXiv:2006.00903v3

[17] Harder, G.: Lectures on algebraic geometry I. sheaves, cohomology of sheaves, and applications to Riemann surfaces. Springer, Wiesbaden (2011)

[18] Hartshorne, R.: Algebraic geometry. Springer, New York (1977)

[19] He, W.: On Calabi's extremal metric and properness. Trans. Am. Math. Soc. 372(8), 5595-5619 (2019)

[20] Hisamoto, T.: On the limit of spectral measures associated to a test configuration of a polarized Kähler manifold. J. Reine Angew. Math. 713, 129-148 (2016)

[21] Hisamoto, T.: Orthogonal projection of a test configuration to vector fields. arXiv: $1610.07158 \mathrm{v} 3$

[22] Hisamoto, T.: Stability and coercivity for toric polarizations. arXiv: 1610.07998v3

[23] Hisamoto, T.: Mabuchi's soliton metric and relative D-stability. arXiv: 1905.05948v2

[24] Huybrechts, D.: Complex geometry: an introduction. Springer, Berlin (2005)

[25] Lazarsfeld, R.: Positivity in algebraic geometry I: Classical setting: line bundles and linear series. Springer, Berlin (2004)

[26] Lazarsfeld, R., Mustaţă, M.: Convex bodies associated to linear series. Ann. Sci. Éc. Norm. Supér. 42(5), 783-835 (2009)

[27] Li, C.: G-uniform stability and Kähler-Einstein metrics on Fano varieties. To appear in Invent. Math. https://doi.org/10.1007/s00222-021-01075-9 (2021)

[28] Li, C.: Geodesic rays and stability in the cscK problem. arXiv: 2001.01366

[29] Li, Y., Zhou, B.: Mabuchi metrics and properness of the modified Ding functional. Pacific J. Math. 302(2), 659-692 (2019)

[30] Mabuchi, T.: Kähler-Einstein metrics for manifolds with nonvanishing Futaki character. Tohoku Math. J. $\mathbf{5 3}(2), 171-182(2001)$

[31] Mabuchi, T.: A theorem of Calabi-Matsushima's type. Osaka J. Math. 39, 49-57 (2002)

[32] Mabuchi, T.: Multiplier Hermitian structures on Kähler manifolds. Nagoya Math. J. 170, 73-115 (2003)

[33] Meinrenken, E.: On Riemann-Roch Formulas for Multiplicities. J. Amer. Math. Soc. 9(2), 373-389 (1996)

[34] Nitta,Y., Saito S., Yotsutani, N.: Relative Ding stability of toric Fano manifolds in low dimensions. arXiv: $1712.01131 \mathrm{v} 3$

[35] Okounkov, A.: Brunn-Minkowski inequality for multiplicities. Invent. Math. 125(3), 405-411 (1996)

[36] Phong, D., Sturm, J.: Test configurations for K-stability and geodesic rays. J. Symplectic Geom. 5(2), 221247 (2007)

[37] Ross, J., Thomas, R.: A study of the Hilbert-Mumford criterion for the stability of projective varieties. J. Algebraic Geom. 16(2), 201-255 (2007)

[38] Sjöström Dyrefelt, Z.: K-semistability of cscK manifolds with transcendental cohomology class. J. Geom. Anal. 28(4), 2927-2960 (2018)

[39] Székelyhidi, G.: Extremal metrics and K-stability. Bull. Lond. Math. Soc. 39(1), 76-84 (2007)

[40] Wang, X., Zhu, X.: Kähler-Ricci solitons on toric manifolds with positive first Chern class, Adv. Math. 188(1), 87-103 (2004)

[41] Witt Nyström, D.: Test configurations and Okounkov bodies. Compos. Math. 148(6), 1736-1756 (2012)

[42] Yao, Y.: Mabuchi solitons and relative Ding stability of toric Fano varieties. To appear in Int. Math. Res. Not. IMRN. https://doi.org/10.1093/imrn/rnab226 (2021)

Email address: yeeyoe@163.com 
School of Mathematics, Hunan University, Changsha, 410082, CHINA. 